\title{
On the massive stellar population of the super star cluster Westerlund 1 *
}

\author{
J. S. Clark ${ }^{1}$, I. Negueruela ${ }^{2}$, P. A. Crowther ${ }^{3}$, and S. P. Goodwin ${ }^{4}$ \\ ${ }^{1}$ Department of Physics and Astronomy, University College London, Gower Street, London, WC1E 6BT, UK \\ e-mail: jsc@star.ucl.ac.uk \\ 2 Dpto. de Física, Ingeniería de Sistemas y Teoría de la Señal, Universidad de Alicante, Apdo. 99, E03080 Alicante, Spain \\ 3 Department of Physics and Astronomy, University of Sheffield, Sheffield, S3 7RH, England, UK \\ ${ }^{4}$ Department of Physics and Astronomy, University of Wales, Cardiff, CF24 3YB, Wales, UK
}

Received 22 November 2004 / Accepted 16 January 2005

\begin{abstract}
We present new spectroscopic and photometric observations of the young Galactic open cluster Westerlund 1 (Wd 1) that reveal a unique population of massive evolved stars. We identify $\sim 200$ cluster members and present spectroscopic classifications for $\sim 25 \%$ of these. We find that all stars so classified are unambiguously post-Main Sequence objects, consistent with an apparent lack of an identifiable Main Sequence in our photometric data to $V \sim 20$. We are able to identify rich populations of Wolf Rayet stars, OB supergiants and short lived transitional objects. Of these, the latter group consists of both hot (Luminous Blue Variable and extreme B supergiant) and cool (Yellow Hypergiant and Red Supergiant) objects - we find that half the known Galactic population of YHGs resides within Wd 1. We obtain a mean $V-M_{V} \sim 25$ mag from the cluster Yellow Hypergiants, implying a Main Sequence turnoff at or below $M_{V}=-5$ (O7 V or later). Based solely on the masses inferred for the 53 spectroscopically classified stars, we determine an absolute minimum mass of $\sim 1.5 \times 10^{3} M_{\odot}$ for $\mathrm{Wd} 1$. However, considering the complete photometrically and spectroscopically selected cluster population and adopting a Kroupa IMF we infer a likely mass for $\mathrm{Wd} 1$ of $\sim 10^{5} M_{\odot}$, noting that inevitable source confusion and incompleteness are likely to render this an underestimate. As such, Wd 1 is the most massive compact young cluster yet identified in the Local Group, with a mass exceeding that of Galactic Centre clusters such as the Arches and Quintuplet. Indeed, the luminosity, inferred mass and compact nature of Wd 1 are comparable with those of Super Star Clusters - previously identified only in external galaxies - and is consistent with expectations for a Globular Cluster progenitor.
\end{abstract}

Key words. stars: evolution - open clusters and associations: individual: Westerlund 1 - galaxies: starburst

\section{Introduction}

Massive stars play an important role in the ecology of galaxies, providing a major source of ionising UV radiation, mechanical energy and chemical enrichment (Smith 2005). However, serious gaps in our understanding of massive stars exist throughout their lifecycle, in large part due to the rapid evolution and hence rarity - of such objects. For example, the lack of accurate observational constraints on the metallicity dependant mass loss rates of such stars - particularly for short lived phases such as Luminous Blue Variables (LBVs) and Yellow Hypergiants (YHGs) - restricts our ability to follow their postMain Sequence (MS) evolution. In particular, currently we cannot predict what path a star of given initial mass will follow as it evolves from the Main Sequence through the Wolf Rayet star (WR) phase to supernova (e.g., the "Conti" scenario; Conti 1976; Maeder \& Conti 1994). Clearly, the most direct way to address such problems is to identify and study massive stars

^ Based on observations collected at the European Southern Observatory, La Silla, Chile (ESO 67.D-0211 and 69.D-0039). within clusters, since this enables us to study a coeval population at a uniform metallicity, where the MS turnoff mass and hence post-MS progenitor mass may be accurately determined (e.g., Massey et al. 2001).

Moreover, the study of clusters is important to the wider galactic evolution, since it is generally thought that massive stars form preferentially (perhaps exclusively) in star clusters (e.g., de Grijs 2005). An extreme example of this phenomenon is observed in starburst galaxies, with star formation occurring in Super Star Clusters (SSCs), which are inferred to be several orders of magnitude more massive than galactic open clusters. Such objects may have very different basic properties from their smaller relatives, with many authors arguing for different kinds of top-heavy mass distributions (e.g., M 82-F; Smith \& Gallagher 2001). The relevance of such a possibility is clear when one considers that a large fraction of the stellar population of galaxies may have formed in starburst episodes in the distant past. If such episodes, in which very large numbers of stars form, are in any way fundamentally different from the much more modest star formation episodes that we observe in 
the present Milky Way, our modelling of the history and evolution of galaxies could be profoundly biased.

Unfortunately, dense clusters of massive stars are rare in the galaxy. The Arches \& Quintuplet Galactic Centre clusters (with masses of $\sim 1 \times 10^{4} M_{\odot}$; Figer 2005) are the most massive known; however, observations are hampered by their extreme reddening. Recently, observations of the apparently unremarkable cluster Westerlund 1 (henceforth $\mathrm{Wd} 1$ ) have demonstrated that for over 4 decades the astronomical community has possibly overlooked the presence of a SSC in our own galaxy.

Discovered by Westerlund (1961), photometric (Borgman et al. 1970; Lockwood 1974; Koornneef 1977) and spectroscopic (Westerlund 1987; West87) surveys suggested the presence of a population of highly luminous early and late type supergiants. However, despite these observations Wd 1 languished in relative obscurity throughout the past decade, due in large part to the significant reddening $\left(A_{\mathrm{v}} \sim 12.9 \mathrm{mag}\right.$, Piatti et al. 1998) which made high resolution spectroscopic observations difficult.

Motivated by the unusual radio and mid-IR properties of two cluster members (Clark et al. 1998), we have undertaken an extensive program of spectroscopic and photometric observations of $\mathrm{Wd} \mathrm{1.} \mathrm{The} \mathrm{first} \mathrm{results} \mathrm{of} \mathrm{this} \mathrm{program} \mathrm{-} \mathrm{the} \mathrm{dis-}$ covery of an unexpected large population of WRs and a new LBV - have already been reported in Clark \& Negueruela (2002, 2004). Here we report on the remainder of the observations, specifically their use in constraining the properties of the population of massive post MS objects within Wd 1.

The paper is therefore structured as follows. In Sect. 2 we briefly describe the observations and the data reduction techniques employed. Sections 3 and 4 are dedicated to the presentation and analysis of both spectroscopic and photometric datasets. Finally, we present an analysis of the global properties of the cluster in Sect. 5 and summarise the results in Sect. 6.

\section{Observations and data reduction}

Low resolution spectroscopy of cluster members over the red/near-IR spectral region ( 6000 to $11000 \AA)$ was taken on 2001 June 23rd to 25th from the ESO $1.52 \mathrm{~m}$ telescope at La Silla Observatory, Chile. The telescope was equipped with the Boller \& Chivens spectrograph, the Loral \#38 camera and the \#1 (night 1) and \#13 (nights 2 and 3) gratings, giving dispersions of $\sim 5 \AA /$ pixel and $\sim 7 \AA /$ pixel - leading to resolutions of $\approx 11 \AA$ and $\approx 16 \AA$ - respectively.

Intermediate-resolution spectroscopy of the brighter cluster members was carried out on 2002 June 7th, using the ESO Multi-Mode Instrument (EMMI) on the $3.6 \mathrm{~m}$ New Technology Telescope (NTT) at La Silla. The Red Medium Resolution mode was used. The instrument was equipped with gratings \#6 and \#7 in the red arm and the new $2048 \times 4096$ MIT CCD mosaic (in the $2 \times 2$ binning mode). Grating \#7 covered the $\lambda \lambda 6310-7835 \AA$ region at a dispersion of $\sim 0.8 \AA /$ pixel, while grating \#6 covered the $\lambda \lambda 8225-8900 \AA$ range at $\sim 0.4 \AA /$ pixel.

Data pre-reduction was carried out with MIDAS software, while image processing and reduction were accomplished with the Starlink packages CCDPACK (Draper 2000) and FIGARO (Shortridge 1997).
Finally photometric observations of the field containing Wd 1 were made with the SUperb-Seeing Imager 2 (SuSI2) direct imaging camera on the NTT; the 2 mosaiced $2 \mathrm{k} \times 4 \mathrm{k}$ CCDs providing a $5.5 \times 5.5$ arcmin field of view. Broadband $U B V R I^{1}$ images were obtained in service time on 2001 August 21, with integration times for individual frames ranging from $1200 \mathrm{~s}$ ( $U$ band) to $2 \mathrm{~s}$ ( $R$ and $I$ bands). Given the significant interstellar reddening anticipated for Wd 1 a selection of nearby red Landolt (1992) standards was observed. Debiasing and flat fielding (both dome and twilight flat field frames were obtained) were accomplished with the STARLINK package KAPPA. Final photometry was kindly extracted for us by Peter Stetson, using the DAOPHOT package in IRAF $^{2}$ (Stetson 1991).

\section{Spectroscopic classification}

In total, 27 stars were observed at intermediate resolution between 6500 and $7900 \AA$ and 8200 and $8900 \AA$, and an additional 26 stars - including the 11 WRs identified in Clark \& Negueruela (2002) - were observed at low resolution between 6500 and $8900 \AA$. A finding chart for all spectroscopically observed stars is presented in Fig. 1. Spectra are presented in Figs. 2 (new WR candidates; Sect. 3.1), 3-7 (Super- and Hyper-giants; Sect. 3.2-3.5) and 8 (the unusual sgB[e] star W9; Sect. 3.3.1).

The 6500 to $9000 \AA$ window offers the prospect of spectral classification of moderately reddened objects for which traditional optical criteria are unavailable. Given the presence of transitions from H I, He I and numerous low - e.g., Fe I, Ca II and N I - and high - e.g., He II, C III and N IV - ionisation species, it would appear possible to construct an accurate classification scheme. While authors have recognised the importance of this window (e.g., Andrillat et al. 1995), comparatively few observations exist within the literature, particularly of high luminosity and/or early type stars. We were therefore forced to adopt a twin approach for spectral classification of our spectra - comparison to observational data where possible (spectral type mid-B $\rightarrow \mathrm{G}$ ) supplemented by construction of a grid of synthetic spectra where required (OB supergiants; Appendix A).

The results of the spectral classification are presented in Table 1, along with the original classification by West 87 . Where possible we have adopted the nomenclature of West87; if an individual object is resolved into two or more stars in our data we adopt the convention of retaining the designation of West87, with the addition of the suffix $a$ for the brightest component, $b$ for the second brightest etc. We further present the $\mathbf{J} 2000$ co-ordinates and the $B V R I$ broadband photometry for each object if available.

Note that while we have utilised a grid of synthetic spectra for the classification of the OB star population of $\mathrm{Wd} 1$ we

\footnotetext{
1 The $U$ band frames produced no useable photometry of cluster members due the to high reddening towards Wd 1 (Sect. 4) and so are not discussed further.

2 IRAF is distributed by the National Optical Astronomy Observatories, which are operated by the Association of Universities for Research in Astronomy, Inc., under cooperative agreement with the National Science Foundation.
} 


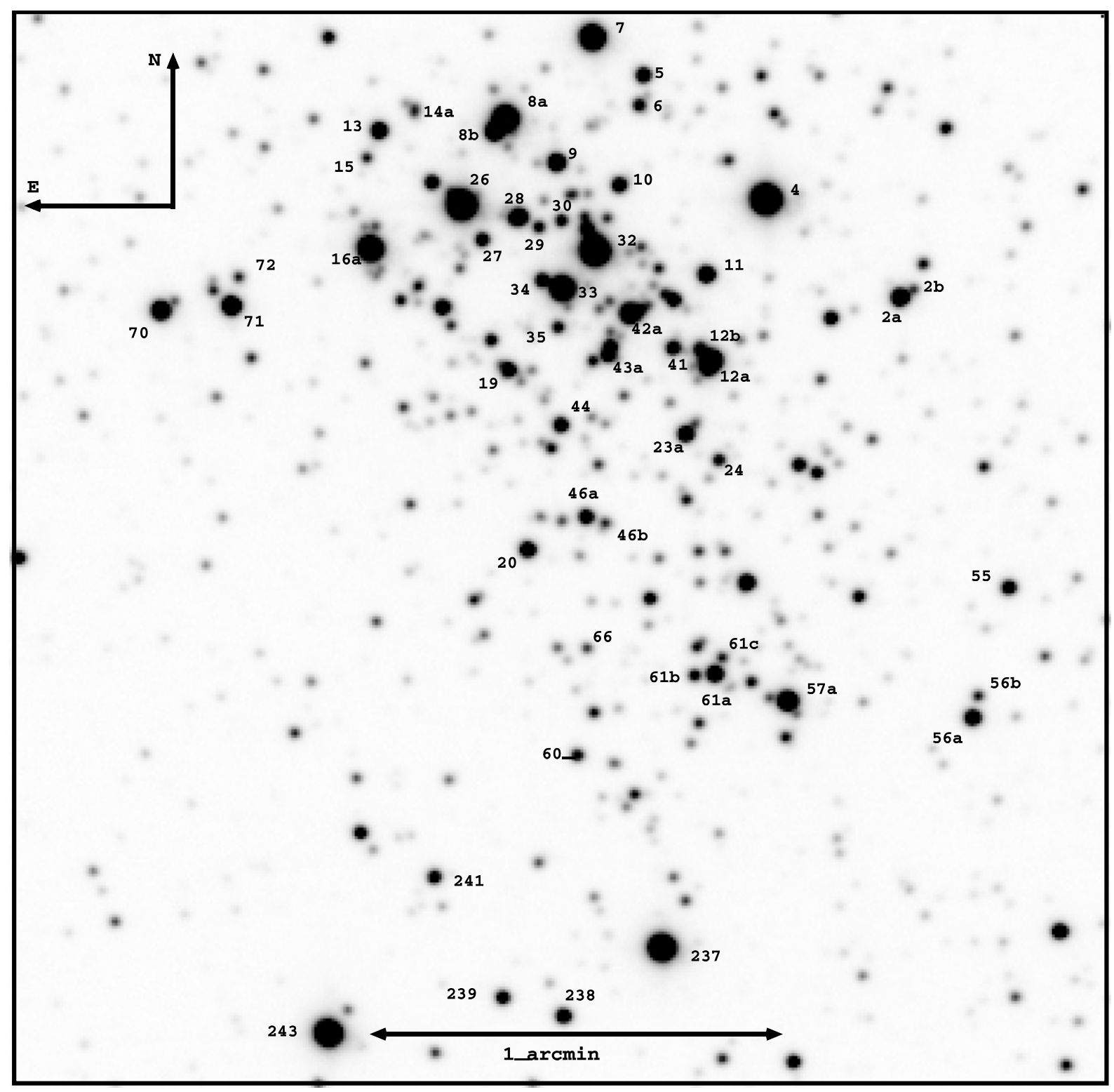

Fig. 1. $I$ band finding chart for Wd 1. Several additional objects have also been indicated where unclear in Fig. 1 of West87; W31 has not been labelled due to crowding, but is the blended object contiguous with and to the north of, W32, while W43a and W42a are the brighter, southernmost components of their respective blends.

do not claim that these represent tailored analyses of individual stars. Given the breadth and complexity of the spectra presented, quantitative analysis is clearly beyond the scope of the current work, which aims to provide coarse spectral classifications for the objects presented.

\subsection{The candidate Wolf Rayet stars}

Clark \& Negueruela (2002) reported the serendipitous discovery of $11 \mathrm{WR}$ stars within $\mathrm{Wd} 1$ in the low resolution 2001 ESO $1.52 \mathrm{~m}$ dataset, comprising $6 \mathrm{WN}$ and $5 \mathrm{WC}$ stars. Analysis of the 2002 intermediate resolution NTT data reveals a further two candidates within the cluster. These are W44 and W66 (=Candidates L and M respectively, following the notation of Clark \& Negueruela 2002); 6500 to $7900 \AA$ spectra for both stars are presented in Fig. 2.
Turning first to W66, Fig. 2 reveals prominent blended emission in He II 6560+C II $6578 \AA$, C II 6725-42+

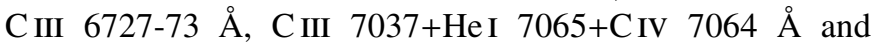
C II $7236+C$ III $7210-12 \AA$. Difficulty in defining a continuum shortwards of $7000 \AA$ prevents the accurate measurement of line strengths for the first 3 blends, but we measure an $E W$ $-130 \pm 15 \AA$ for the $\mathrm{C}_{\text {II }} 7236+\mathrm{C}_{\text {III }} 7210-12 \AA$ blend. For the lowest excitation WC8 star (WR 53), the C IV $7065 \AA$ and C II $7236 \AA$ lines are of similar strength, while all WC9 stars have C II $7236 \AA$ A IV $7065 \AA$, indicating a classification of WC9 for W66.

W44 demonstrates emission in the comparatively low ionisation lines of $\mathrm{H} \alpha(E W \sim-30 \AA)$, He I $6678(E W \sim-8 \AA)$ and $7065(E W \sim-14 \AA)$, with no emission from either higher (e.g., He II, N III-IV or C III-IV) or lower ionisation species (e.g., Fe II). The spectrum in the 8200 to $8900 \AA$ range appears 


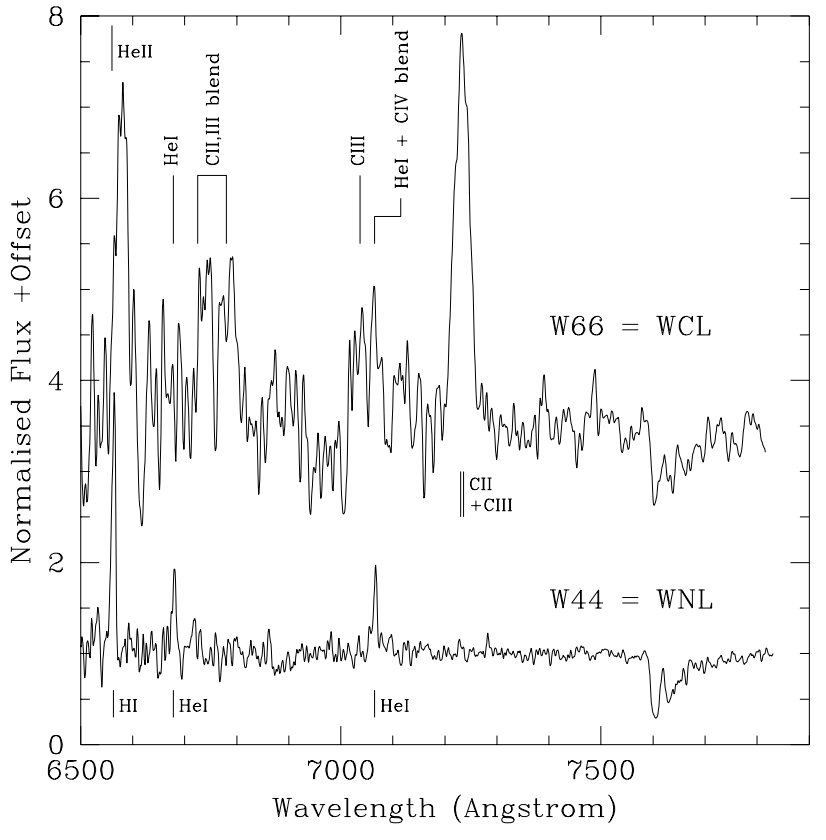

Fig. 2. $R$ band spectra of the 2 newly discovered WR stars in the cluster; Candidate L $(=\mathrm{W} 44)$, a probable WN9 star and Candidate $\mathrm{M}$ (=W66), a sixth WCL star (WC9).

completely featureless (to a limit of $E W \pm 0.5 \AA$ ) and hence is not presented. Given the apparent magnitude of W44, combined with the strength of the emission lines, the lack of Paschen lines (emission or absorption) and low ionisation species, we exclude a B emission line star classification - such as classical $\mathrm{Be}$ or $\mathrm{sgB}[\mathrm{e}] \mathrm{star}$ - and instead suggest a WN identification. The lack of N IV $7116 \AA$ precludes a classification earlier than WN9, with a classification of WN9 rather than WN9ha more likely due to the comparative strength of the HeI lines. This is the first such star identified within Wd 1, although we note that the low resolution spectrum of W5 is possibly indicative of an even later WNL classification - this is detailed in Sect. 3.3.3.

\subsection{The late $O$ and early- $B$ supergiants}

Intermediate resolution spectra of all 13 stars in Fig. 4 and W7, 33, 70 and 71 in Fig. 5 fulfill the criteria for OB spectral types defined in Appendix A and individual classifications are listed in Table 1 . The FWHM of the Paschen lines clearly satisfy the criterion of Caron et al. (2003) for supergiants, forming a continuous sequence with the later A-F stars in Fig. 5, which we demonstrate in Sect. 3.4 to also be super- or hyper-giants.

We find W57a, 70 and 71 to have the latest spectral type B3 Ia - of the continuous sequence of OB supergiants present in the sample. This classification may be assigned on the basis of the presence and strength of weak N I and O I $8446 \AA$ absorption. While W7 and 33 apparently are of later spectral type, their spectra demonstrate several unusual properties that suggest they have more in common with the transitional objects such as the LBV W243 and the YHGs; hence we defer discussion until Sect. 3.3.
The remaining spectra display solely $\mathrm{HI}$ and He I photospheric absorption lines, implying subtypes of B1.5 Ia or earlier $\left(T_{\text {eff }} \geq 20 \mathrm{kK}\right)$. In addition, we find no signature of the He II $8238 \AA$ line in any object, suggesting subtypes no earlier than O9.5 Ia. Further subclassification is hampered by the poor $\mathrm{S} / \mathrm{N}$ of most spectra, which precludes the use of the $\mathrm{Pa} 16+\mathrm{C}$ III/Pa15 line ratio.

Consequently, we may only attempt additional classification based on the strengths of the Paschen lines. Measuring the strengths of the Paschen lines in the observed (and synthetic) spectra relative to W70 and 71 (B3 Ia model) suggests an approximate range of spectral types for W61a, 60, 43a, 19, 23a, $11,2 \mathrm{a}$ and 28 (earliest $\rightarrow$ latest) of O9.5 Ia $\rightarrow$ B 0.5 Ia ( $30 \mathrm{kK} \rightarrow$ $24 \mathrm{kK})$. While the systematic weakening in line strength - resulting in the absence of higher Paschen series lines - of stars such as W61a and 60 compared to W2a and 28 is clearly indicative of graduations in spectral type within this group, we refrain from attempting any further subclassification based on current data.

Finally, while the spectra of W30,13, 61b and 24 are almost completely devoid of photospheric features and hence suggest spectral types of O9 Ia or earlier, the absence of He II $8238 \AA$ apparently precludes such a possibility. Instead we consider it likely that possible binarity or blending of 2 nearby objects leads to the featureless spectra and hence refrain from a classification other than generic early OB supergiant for these 4 objects.

Although the $\mathrm{S} / \mathrm{N}$ for many spectra is rather poor, we find no evidence for a photospheric $\mathrm{H} \alpha$ profile as predicted by our synthetic spectra in the temperature range 10-34 kK. A montage of the region containing the $\mathrm{H} \alpha$ line for those spectra with the largest $\mathrm{S} / \mathrm{N}$ is presented in Fig. 6; we find evidence for either infilling (W19, 23a, 24, 43a, 57a 60, 61b and 70) or emission (W11, 13, 28, 30, 61a and 71) in all spectra. Preempting the results of Sect. 4, in the absence of an accurate photometric determination of bolometric luminosity, we may use the presence of $\mathrm{H} \alpha$ emission to infer a large luminosity and initial mass for the OB supergiants. Specifically, McErlean et al. (1999) show that of their sample all the most luminous $(M \sim$ 30-40 $M_{\odot}$ ) B supergiants demonstrate $\mathrm{H} \alpha$ emission.

Figure 7 presents a montage of the low resolution spectra of OB star candidates; where intermediate resolution spectra exist, these have been overplotted. Given both the lower resolution and $\mathrm{S} / \mathrm{N}$ of the spectra, detailed classification is difficult. Hence we simply choose to divide the spectra into three morphological groups based on the presence of various photospheric features. Group 1 contains those stars without an obvious Paschen series and consists of W13,14a, 15 and 44. Group 2 contains those stars with the Paschen series in absorption and consists of W2a, 5, 6, 10, 11, 23a, 24, 29, 43a, 55, 56 and 238. Finally, group 3 consists of those stars with both Paschen and the O I $7774 \AA$ blend in absorption, and consists of $\mathrm{W} 7,33,41,42,70$ and 71.

Of the group 1 objects W44 and W13 have already been discussed. With an essentially featureless spectrum we suspect that W15 is likely a binary or blend of one or more OB SGs. The strong N IV emission seen in W14a is unexpected since this would imply an early $\mathrm{O}$ supergiant classification, at odds 
Table 1. Summary of the 53 cluster members with spectroscopic classifications. Column 1 provides the notation of West87, while Col. 2 provides our additional notation if required. Columns 3 and 4 provide the Equ J2000 co-ordinates, while broadband BVRI photometric values are presented in Cols. 5-8 where available. Columns 9 and 10 list spectral classifications from West 87 and this work, respectively. Classifications listed in italics were derived from low resolution spectra. In general we find an encouraging correspondence between the two works, although our classifications appear to be systematically earlier for those stars classified as late B-early G by West87. Importantly we confirm the classification of West87's four YHG candidates, while identifying a further 2 candidates. Finally, we highlight one important difference; we are unable to identify any Main Sequence or giant stars, with all the OB stars in our study being classified as Supergiants (W6, 10, 11, 28, 33, 70 and 71 receiving revised luminosity classes).

\begin{tabular}{|c|c|c|c|c|c|c|c|c|c|}
\hline W87 & $\begin{array}{c}\mathrm{ID} \\
(\mathrm{new})\end{array}$ & RA (J2000) & 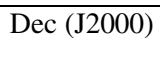 & $\overline{\bar{B}}$ & $\overline{V V}$ & $\bar{R}$ & $\overline{I I}$ & $\begin{array}{c}\text { Spectral } \\
\text { W87 }\end{array}$ & $\begin{array}{l}\text { Type } \\
\text { New }\end{array}$ \\
\hline 2 & $2 a$ & 164659.71 & -455051.1 & 20.4 & 16.69 & 14.23 & 11.73 & - & O9.5 Ia - B0.5 Ia \\
\hline 4 & & 164701.42 & -455037.1 & 18.7 & 14.47 & 11.80 & 9.15 & $\mathrm{G} 0 \mathrm{Ia}^{+}$ & $\mathrm{F} 2 \mathrm{Ia}^{+}$ \\
\hline 5 & & 164702.97 & -455019.5 & 21.4 & 17.49 & 14.98 & 12.48 & - & WNL/early $\mathrm{B} \mathrm{Ia}^{+}$ \\
\hline 6 & & 164703.04 & -455023.6 & 22.2 & 18.41 & 15.80 & 13.16 & $\mathrm{~B} 1 \mathrm{~V}$ & O9.5 Ia - B0.5 Ia \\
\hline 7 & & 164703.62 & -455014.2 & 20.0 & 15.57 & 12.73 & 9.99 & A0 Ia & B5 $\mathrm{Ia}^{+}$ \\
\hline 8 & $8 a$ & 164704.79 & -455024.9 & 19.9 & 15.50 & 12.64 & 9.89 & G0 Ia & $\mathrm{F} 5 \mathrm{Ia}^{+}$ \\
\hline 9 & & 164704.14 & -455031.1 & 21.8 & 17.47 & 14.47 & 11.74 & $\mathrm{~B}[\mathrm{e}]$ & $\operatorname{sgB}[\mathrm{e}]$ \\
\hline 10 & & 164703.32 & -455034.7 & - & - & - & - & B0 III & O9.5 Ia - B0.5 Ia \\
\hline 11 & & 164702.23 & -455047.0 & 21.2 & 17.15 & 14.52 & 11.91 & B1 II & O9.5 Ia - B0.5 Ia \\
\hline 12 & $12 \mathrm{a}$ & 164702.21 & -455058.8 & 22.0 & 16.94 & 13.54 & 10.42 & $\mathrm{~A} 2 \mathrm{Ia}^{+}$ & $\mathrm{A} 5 \mathrm{Ia}^{+}$ \\
\hline 13 & & 164706.45 & -455026.0 & 21.1 & 17.19 & 14.63 & 12.06 & - & OB binary/blend? \\
\hline 14 & $14 \mathrm{a}$ & 164705.94 & -45 5023.3 & - & - & - & - & - & OB+WN blend? \\
\hline 15 & & 164706.63 & -45 5029.7 & 22.8 & 18.96 & 16.38 & 13.75 & - & OB binary/blend? \\
\hline 16 & $16 \mathrm{a}$ & 164706.61 & -455042.1 & 20.5 & 15.89 & 12.82 & 9.90 & A $2 \mathrm{Ia}^{+}$ & A $2 \mathrm{Ia}^{+}$ \\
\hline 19 & & 164704.86 & -455059.1 & 22.6 & 18.22 & 15.21 & 12.37 & - & O9.5 Ia - B0.5 Ia \\
\hline 20 & & 164704.70 & -455123.8 & - & - & - & - & M6 I & $<M 6 I$ \\
\hline 23 & $23 a$ & 164702.57 & -455108.7 & 22.1 & 17.85 & 14.91 & 12.07 & - & O9.5 Ia - B0.5 Ia \\
\hline 24 & & 164702.15 & -455112.4 & 23.0 & 18.71 & 15.96 & 13.24 & - & OB binary/blend? \\
\hline 26 & & 164705.40 & -455036.5 & 22.1 & 16.79 & 12.63 & 9.19 & M2 I & $<M 6 I$ \\
\hline 28 & & 164704.66 & -455038.4 & 20.9 & 16.87 & 14.26 & 11.64 & B0 II & O9.5 Ia - B0.5 Ia \\
\hline 29 & & 164704.41 & -455039.8 & 22.6 & 18.66 & 16.02 & 13.38 & - & O9.5 Ia - B0.5 Ia \\
\hline 30 & & 164704.11 & -455039.0 & 22.4 & 18.45 & 15.80 & 13.20 & - & OB binary/blend? \\
\hline 32 & & 164703.67 & -455043.5 & - & - & - & - & G5 Ia & $\mathrm{F} 5 \mathrm{Ia}^{+}$ \\
\hline 33 & & 164704.12 & -455048.3 & 20.0 & 15.61 & 12.78 & 10.04 & B8 I & B5 $\mathrm{Ia}^{+}$ \\
\hline 35 & & 164704.20 & -455053.5 & 22.7 & 18.59 & 16.00 & 13.31 & - & O9.5 Ia - B0.5 Ia \\
\hline 41 & & 164702.70 & -455056.9 & 21.3 & 17.87 & 15.39 & 12.78 & - & OB binary/blend? \\
\hline 42 & $42 \mathrm{a}$ & 164703.25 & -455052.1 & - & - & - & - & - & $\mathrm{BS}_{\mathrm{Ia}}^{+}$? \\
\hline 43 & $43 a$ & 164703.54 & -455057.3 & 22.8 & 18.05 & 15.22 & 12.26 & - & O9.5 Ia - B0.5 Ia \\
\hline 44 & $\mathrm{~L}$ & 164704.20 & -455106.9 & 22.6 & 18.86 & 15.61 & 12.52 & - & WN9 \\
\hline 55 & & 164658.40 & -455131.2 & 21.6 & 17.67 & 15.25 & 12.67 & - & O9.5 Ia - B0.5 Ia \\
\hline 56 & & 164658.93 & -455148.8 & 21.7 & 17.46 & 14.81 & 12.15 & - & O9.5 Ia - B0.5 Ia \\
\hline 57 & $57 \mathrm{a}$ & 164701.35 & -455145.6 & 20.7 & 16.54 & 13.83 & 11.13 & - & B3 Ia \\
\hline 60 & & 164704.13 & -455152.1 & 22.8 & 18.50 & 15.96 & 13.28 & - & O9.5 Ia - B0.5 Ia \\
\hline \multirow[t]{2}{*}{61} & $61 \mathrm{a}$ & 164702.29 & -455141.6 & 21.2 & 17.16 & 14.62 & 12.01 & - & O9.5 Ia - B0.5 Ia \\
\hline & $61 b$ & 164702.56 & -455141.6 & 22.7 & 18.59 & 16.00 & 13.31 & - & OB binary/blend? \\
\hline 66 & M & 164703.96 & -455137.5 & - & 19.79 & 16.85 & 13.96 & - & WC9 \\
\hline 70 & & 164709.36 & -455049.6 & 21.2 & 16.88 & 14.10 & 11.29 & B8 Iab & B3 Ia \\
\hline 71 & & 164708.44 & -455049.3 & 21.5 & 17.01 & 14.06 & 11.16 & B8 Iab & B3 Ia \\
\hline 72 & A & 164708.32 & -455045.5 & - & 19.69 & 16.59 & 13.68 & - & WN4-5 \\
\hline 237 & & 164703.09 & -455218.8 & 22.8 & 17.49 & 13.00 & 9.40 & M6+ III & $<M 6 I$ \\
\hline 238 & & 164704.41 & -455227.6 & 21.4 & 17.47 & 14.98 & 12.45 & - & O9.5 Ia - B0.5 Ia \\
\hline 239 & $\mathrm{~F}$ & 164705.21 & -455225.0 & 21.7 & 17.86 & 15.39 & 12.90 & - & WC9 \\
\hline 241 & E & 164706.06 & -455208.3 & - & - & - & - & - & WC9 \\
\hline 243 & & 164707.55 & -455228.5 & - & - & - & - & B2 Ia & LBV \\
\hline 265 & & 164706.26 & -45 4923.7 & 22.0 & 17.05 & 13.62 & 10.54 & $\mathrm{G} 0 \mathrm{Ia}^{+}$ & $\mathrm{F} 5 \mathrm{Ia}^{+}$ \\
\hline - & B & 164705.35 & -455105.0 & - & 20.99 & 17.50 & 14.37 & - & WN6-8 \\
\hline - & $\mathrm{C}$ & 164704.40 & -455103.8 & - & - & - & - & - & WC8 \\
\hline - & D & 164706.24 & -455126.5 & - & - & - & - & - & WN6-8 \\
\hline - & G & 164704.02 & -455125.2 & 22.7 & 20.87 & 17.75 & 14.68 & - & WN6-8 \\
\hline - & $\mathrm{H}$ & 164703.91 & -455119.9 & 23.0 & 18.55 & 15.46 & 12.46 & - & WC9 \\
\hline- & I & 164701.67 & -455120.4 & - & - & - & - & - & WN6-8 \\
\hline- & $\mathrm{J}$ & 164700.89 & -455120.9 & - & - & - & - & - & WN6-8 \\
\hline - & K & 164702.70 & -455057.4 & - & - & - & - & - & WC8-9 \\
\hline
\end{tabular}



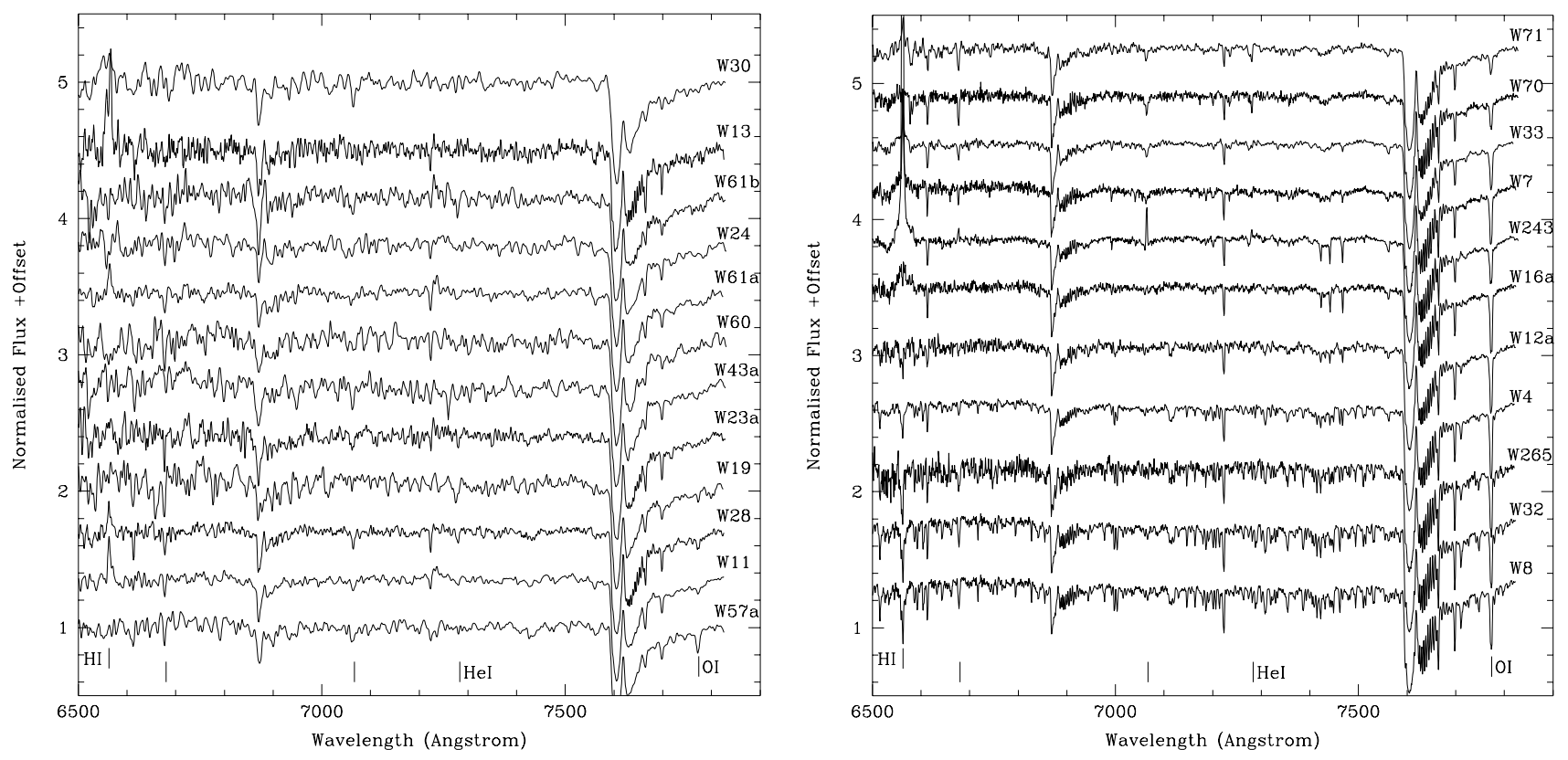

Fig. 3. Intermediate resolution $R$ band (6400 to $7800 \AA$ ) spectroscopy of O-F supergiant and hypergiant members of Wd 1 . Prominent transitions of HI, He I and O I are indicated. Left panel: O and mid B supergiants; we find no evidence for He II absorption in the spectra. Right panel: mid-B to F supergiants in Wd 1 . Weak C II $6578 / 82 \AA$ absorption is visible in the red wing of the $\mathrm{H} \alpha$ line in the mid-B supergiants. We tentatively identify the three prominent absorption features between $7420-7450 \AA$ in W243 and W16a as N I 7423.6, 7442.3 and $7468.3 \AA$. Weak Fe I lines at 7112.2 and $6999.9 \AA$ also appear to be present in the spectrum of W12a $\left(\mathrm{A}_{5} \mathrm{Ia}^{+}\right)$and later objects. Possible identifications of the absorption features in the mid-A and later spectral types at $\sim 7711 \AA$ and $\sim 7748 \AA$ are Fe II 7712 and Fe I $7748.3 \AA$ respectively.

with the expected age of the cluster (Sect. 5). However, we note that W14a appears to be composed of 2 or more stars; hence an alternative explanation would be a blend containing at least one hitherto undetected WNE star. Clearly, future observations are required to resolve this apparent inconsistency.

With a lack of O I $7774 \AA$ absorption, the group 2 objects appear to have earlier spectral types than $\sim$ B3 Ia. Indeed all those objects for which intermediate resolution observations are available are of spectral type O9.5 Ia to B0.5 Ia - hence we assume a late $\mathrm{O} /$ early $\mathrm{B}$ supergiant classification for the remaining objects, with the sole exception of W5, which, together with W7 and 33, we discuss in Sect. 3.3.3.

Of the group 3 spectra W41 and 42 have only been observed at low resolution. W41 apparently shows weak O I absorption, although it is coupled with an unexpectedly weak Paschen series. $\mathrm{H} \alpha$ is clearly seen in absorption; the only such object in our dataset. Given the rather unusual nature of the spectrum we tentatively suggest the spectrum may be a blend of two or more stars. By contrast W42a has pronounced $\mathrm{H} \alpha$ emission and appears similar to the mid B supergiants W7 and 33 (Sect. 3.3.3) and is discussed therein.

Finally, comparison of the two epochs of observations of W23a and 43a are suggestive of variability, with $\mathrm{H} \alpha$ emission conspicuous in 2001, but apparently absent in 2002 .

\subsection{Anomalous $B$ supergiants}

Amongst the observations of the OB supergiants within the cluster we find a significant number of objects with spectra demonstrating unusual properties. In this subsection we detail these individually.

\subsubsection{The $\mathrm{sgB}[\mathrm{e}]$ star $\mathrm{W} 9$}

The spectrum of W9 is presented in Fig. 8 and is dominated by $\mathrm{HI}$, He I and low ionisation metallic emission (Fe II, Ca I, N I and OI), with narrow, single peaked emission lines $\left(F W H M(\mathrm{H} \alpha)=125 \mathrm{~km} \mathrm{~s}^{-1}\right)$ and is notable for the complete lack of any photospheric features and the strength of certain emission features (e.g., $W_{\lambda}(\mathrm{H} \alpha)=-520 \pm 30 \AA, W_{\lambda}(\mathrm{OI})=$ $-48 \pm 2 \AA$ ). To the best of our knowledge such an equivalent width for $\mathrm{H} \alpha$ is completely unprecedented for an early type emission line star, with the exception of the extreme systems SS433 (X-ray binary; e.g., Falomo et al. 1987) and $\eta$ Car (LBV; e.g., Davidson et al. 1999). Radio observations (Clark et al. 1998; Dougherty et al. 2005) reveal a strong, compact radio source to be associated with W9, while mid IR observations likewise show strong emission $\left(F_{10 \mu \mathrm{m}}=50 \mathrm{Jy}\right)$, although the emission mechanism (thermal dust or $\mathrm{f}-\mathrm{f} / \mathrm{b}-\mathrm{f}$ ) is still uncertain. Finally, an ISO-SWS spectrum reveals the presence of [O IV] emission, implying an exciting source with a temperature in excess of $\sim 80 \mathrm{kK}$ must be present (Clark et al. 1998).

While a quantitative analysis of the combined dataset is beyond the scope of this work, we note that the observational properties of W9 are unique within Wd 1, and apparently amongst the wider galactic population of early $\mathrm{OB}$ stars. While it fulfills the classification criteria for supergiant $\mathrm{B}[\mathrm{e}]$ stars of Lamers et al. (1998) we suspect that the emission line spectrum arises at least partially in the compact circumstellar envelope 


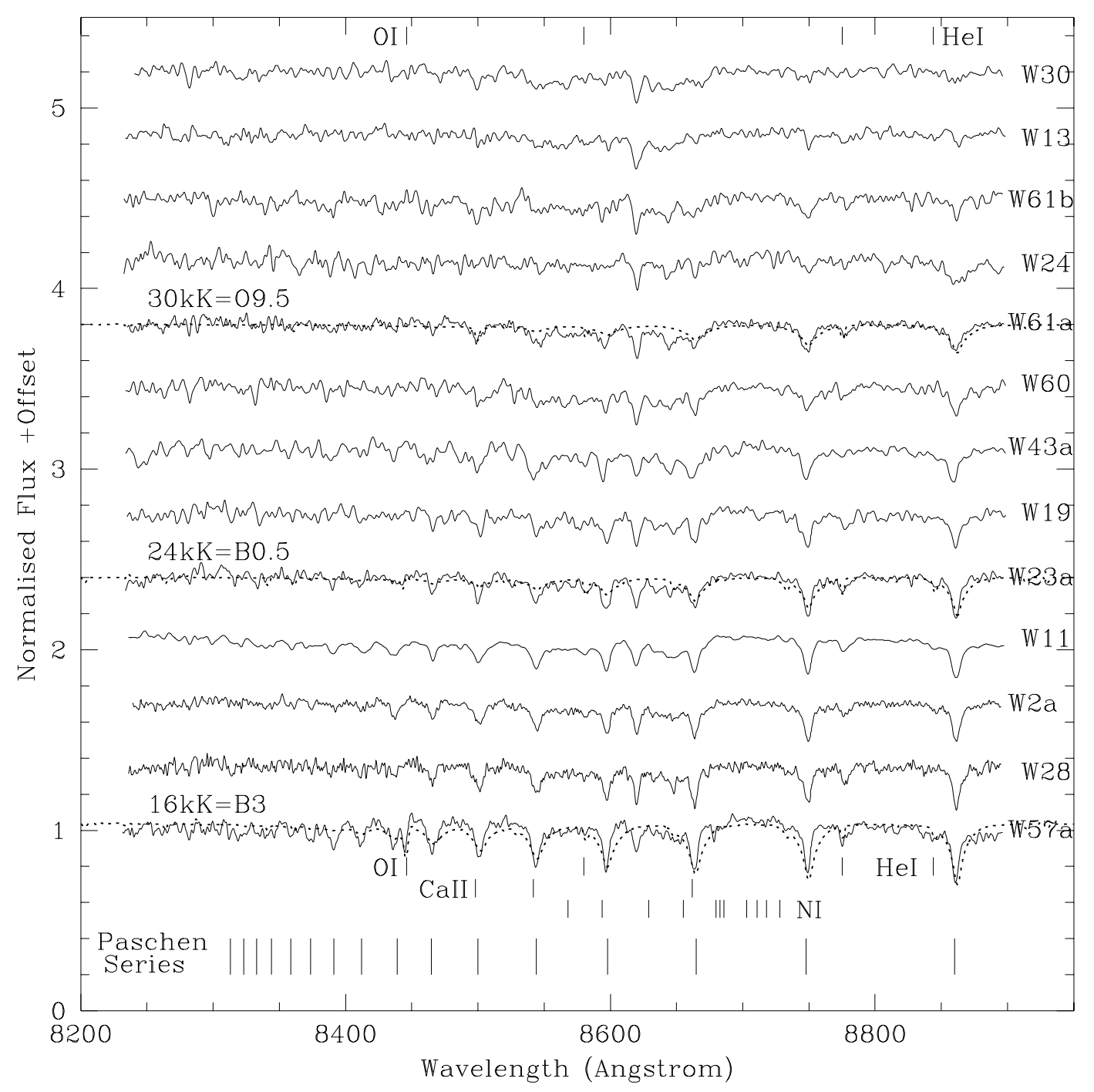

Fig. 4. Intermediate resolution $I$ band (8200 to $8900 \AA$ ) spectroscopy (I): O and B supergiants in Wd 1 . We find the spectra of earliest stars to be almost featureless, with the exception of the strongest Paschen series, while weak He I absorption appears later. In the latest spectral types ( $\sim$ B3) shown in this figure, weak absorption features of O I and N I are also present. Note the feature at $\sim 8620 \AA$ is a D.I.B. Representative synthetic spectra have been overplotted on selected objects - note that these have not been tailored to individual stars and hence we do not claim a formal fit to these data; such a goal is left for a future paper.

rather than in a stellar wind (Clark et al. 1998). As such it could closely resemble the unusual object NaSt 1 , which Crowther \& Smith (1999) propose to consist of a compact ejection nebula surrounding - and obscuring - an exciting WNE object. Such an hypothesis would naturally explain the [O IV] emission present in W9, although one might question the compact nature - and hence implied youth - of the radio nebula when contrasted to other LBV nebulae, given the requirement for the central star to evolve through both the LBV and WNL phases.

Clearly, while such an objection could be overcome by proposing a compact $\mathrm{WR}+\mathrm{LBV}$ binary system, we also suggest an alternative possibility, namely that W9 is the result of a recent merger event. Given the severely crowded nature of the central regions of the cluster (Sect. 5.4) stellar collisions and interactions appear likely. A recent $\left(\sim 10^{3} \mathrm{yr}\right)$ interaction in which the outer $\mathrm{H}$ mantle of one or both objects were lifted off to form the compact radio nebula would reveal the hot interior layers of the star(s) so affected. This would then naturally explain the high excitation emission - until the remnant relaxed to a new equilibrium structure on a thermal timescale $\left(\sim 10^{4} \mathrm{yr}\right)$; a scenario proposed by Clark et al. (2000) to explain the stellar and nebular morphology of the candidate LBV G25.5+0.2.

\subsubsection{The luminous blue variable W243}

Clark \& Negueruela (2004) present multi-epoch data that clearly demonstrate that W243 - initially classified as an early B supergiant by West87 - has evolved in the intervening decades to demonstrate a much cooler, A supergiant spectrum, albeit with anomalous emission features. The overall spectrum closely resembles that of the luminous A supergiant IRC +10 420 (e.g., Oudmaijer 1998), with the notable 


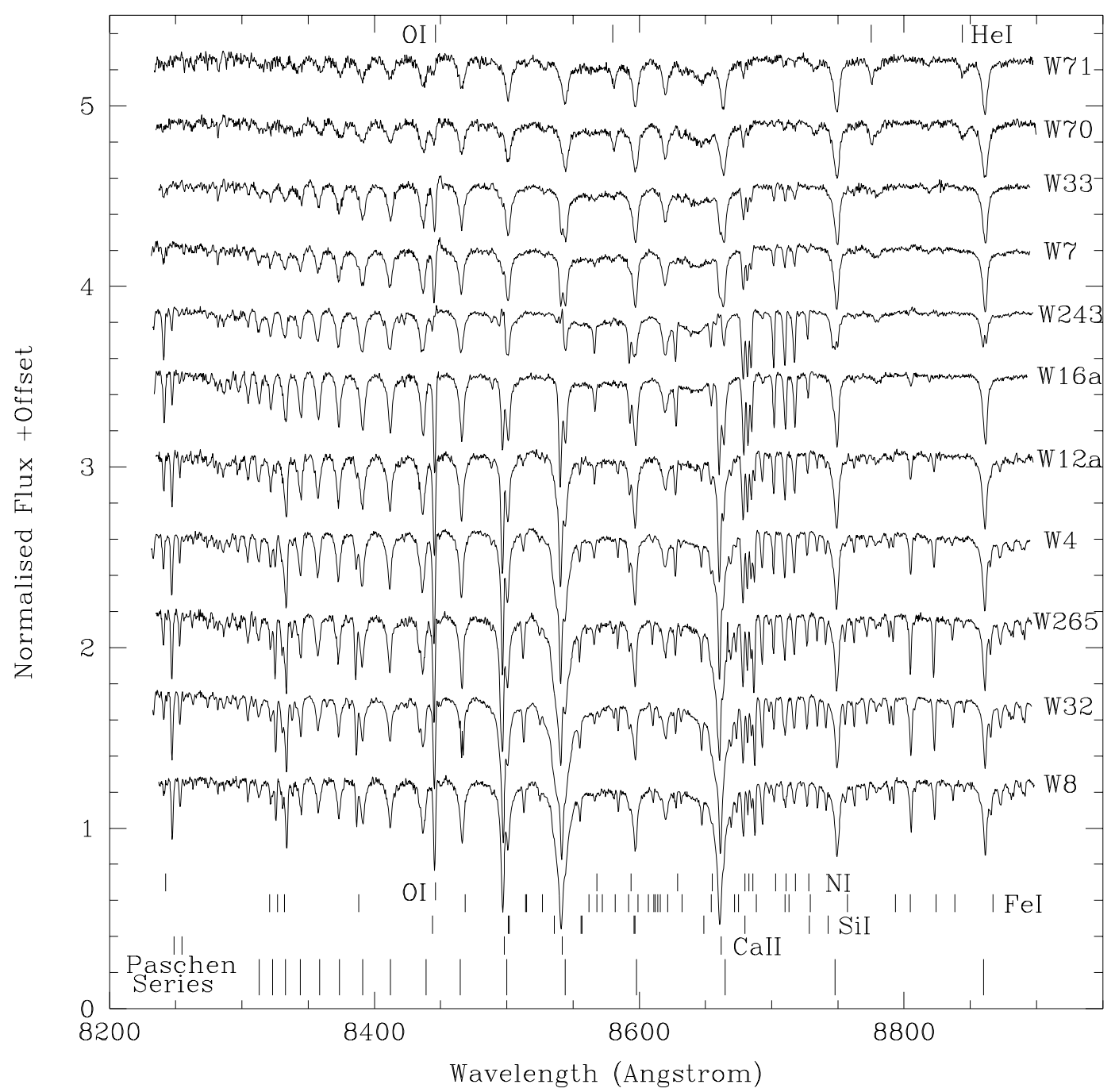

Fig. 5. Intermediate resolution $I$ band ( 8200 to $8900 \AA$ ) spectroscopy (II): mid-B to F supergiants in Wd 1 . All spectra are dominated by the H I Paschen series, while low ionisation species such as Fe I, Si I and particularly N I and Ca II come to dominate the mid-A and later spectra. Despite their presence in the later spectral types transitions of some low ionisation species such as e.g., Mg I identifications are excluded for reasons of clarity; we refer the reader to Fig. 1 of Munari \& Tomaselli (1999) for more complete annotated spectra. Note that we are unable to identify some of the (presumably low ionisation metallic) lines in the later spectral types, notably the strong absorption feature at $\sim 8334 \AA$ (blended with Pa24). While not labelled, the feature at $\sim 8620 \AA$ is a D.I.B.

exception of emission in the He I 6678 and $7065 \AA$ lines. Clark $\&$ Negueruela (2004) advanced the suggestion that W243 was an LBV, with the unusual composite spectrum resulting from the formation of a pseudo-photosphere in a dense stellar wind.

Subsequently, de Koter (2004, priv. comm.) suggested that as presented such an hypothesis would predict significantly stronger $\mathrm{H} \alpha$ emission $(E W \sim-100 \AA)$ than observed. However, the presence of He I emission and strong electron scattering wings (extending to $\pm 10^{3} \mathrm{~km} \mathrm{~s}^{-1}$ ) in the $\mathrm{H} \alpha$ emission profile apparently excludes a simple alternative explanation that W243 now has the physical properties - and in particular a rather low mass loss rate - of a bona fide A supergiant.

Consequently, we suspect that a hybrid model may provide the correct explanation - viz. a cooling of the underlying star to a late B spectral type - still sufficient to produce He I emission coupled with an increase in mass loss rate to simulate a later spectral type.

\subsubsection{Extreme B supergiants $W 5,7,33$ and $42 a$}

Based on the strength of the O I $8446 \AA$ and N I features present in the intermediate resolution spectra (Fig. 5), W7 and 33 appear to be B5 supergiants. However, closer examination shows a weak P Cygni profile in the O I $8446 \AA$ line of both stars - to the best of our knowledge absent from any other mid-B supergiant, with the gnotable exception of the 2002 spectrum of the LBV W243.

Examination of the $\mathrm{H} \alpha$ profiles also shows that they differ from the weak P Cygni profiles expected for B supergiants, being composed of a single narrow peak superimposed on a broad emission base (Fig. 6). In this respect they again resemble the LBV W243 (Fig. 3), while the broad emission component is observed for the cluster member YHG W16a (Sect. 3.4). Moreover, similar composite Balmer line profiles are also obtained for the "B-supergiant LBVs" studied by Walborn \& Fitzpatrick (2000). Consequently, we propose that both objects 

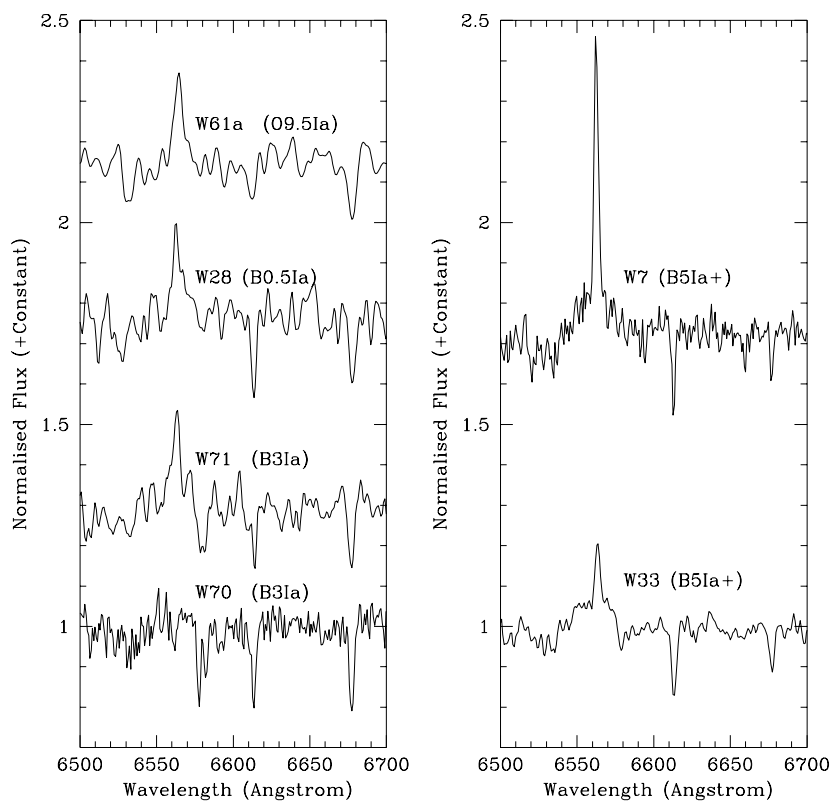

Fig. 6. Montage of the region of the spectrum containing $\mathrm{H} \alpha$ for a selection of the OB supergiants. The left panel shows sample spectra for the O9.5 Ia to B3 Ia stars, the presence of either infilling or $\mathrm{H} \alpha$ emission indicating a Ia luminosity classification. The right panel shows the $\mathrm{H} \alpha$ profiles for the two highly luminous mid-B hypergiants W7 and 33, note the narrow line superimposed on a broader plinth of emission.

are to be found in a shortlived, transitional evolutionary state more closely related to the LBV and YHG population of $\mathrm{Wd} 1$ than the late $\mathrm{O} /$ early $\mathrm{B}$ supergiant population. Indeed, both stars are a magnitude more luminous than any other OB supergiant within the cluster, with an observed $V$ band magnitude directly comparable to the YHG population.

Comparable objects might therefore be the highly luminous B1.5 $\mathrm{Ia}^{+}$hypergiants HD 80077 (Carpay et al. 1991) and HD 152236 (Van Genderen et al. 1984), which are also characterised by strong $\mathrm{H} \alpha$ emission due to mass loss rates $\sim$ an order of magnitude greater than Ia and Ib supergiants of similar spectral type (e.g., Leitherer et al. 1995).

Of the stars for which only low resolution spectra exist, we find that W42a has a comparable morphology to both W7 and 33, suggesting a similar classification. W5 demonstrates both dramatic $\mathrm{H} \alpha$ and also $\mathrm{C}$ II $\sim 7236 \AA$ emission, previously only identified in the WCL population. However, the lack of C III $9705 \AA$ emision (not shown in Fig. 7) precludes an identification as a binary/blend containing a WCL star. Recent high resolution spectra obtained by us (Negueruela \& Clark 2005) confirm the identification of C II emission, while also revealing the presence of weak He I P Cygni emission profiles. Such a morphology, with a lack of N IV 7116 emission, is indicative of either a very late WN star (WN9 or later) or an extreme early $\mathrm{BIa}+$ supergiant, the lack of a He II transition in the current data precluding a further classification.
Table 2. Equivalent widths for the O I $\sim 7774 \AA$ A blend for the six yellow hypergiants in $\mathrm{Wd} 1$, with absolute visual magnitude and resultant luminosity determined directly from the relationship of Arellano Ferro et al. (2003; formal error of $\pm 0.4 \mathrm{mag}$ ).

\begin{tabular}{|c|c|c|c|}
\hline YHG & $\begin{array}{l}\text { Spec. } \\
\text { type }\end{array}$ & $\begin{array}{c}E W(\mathrm{O} \mathrm{I}) \\
(\AA\end{array}$ & $\overline{M_{v}}$ \\
\hline 4 & $\mathrm{~F} 2 \mathrm{Ia}^{+}$ & 3.0 & -10.1 \\
\hline $8 a$ & $\mathrm{~F} 5 \mathrm{Ia}^{+}$ & 2.1 & -8.7 \\
\hline $12 \mathrm{a}$ & A $5 \mathrm{Ia}^{+}$ & 2.8 & -9.9 \\
\hline $16 a$ & $\mathrm{~A} 2 \mathrm{Ia}^{+}$ & 2.7 & -9.8 \\
\hline 32 & $\mathrm{~F} \mathrm{Ia}^{+}$ & 2.3 & -9.1 \\
\hline 265 & $\mathrm{~F} \mathrm{Ia}^{+}$ & 2.6 & -9.7 \\
\hline
\end{tabular}

\subsection{The yellow hypergiants}

The remaining 6 spectra in Figs. 3 and 5 are seen to be dominated by low ionisation metallic photospheric lines, suggesting spectral types later than B. Comprehensive classification criteria and standard spectra for cool supergiants are provided by Munari \& Tomasella (1999). Comparison to their data, employing the relative strengths of the $\mathrm{Ca}$ II lines and adjacent Paschen series lines - providing the same diagnostic as the $\mathrm{Ca}$ II $\mathrm{H}$ and $\mathrm{K}$ versus the Balmer line strength in the optical region achieves a broad classification for B8-F8 supergiants. This may then be further refined by consideration of the presence and strength of the N I transitions between 8650 to $8750 \AA$ for A supergiants and the appearance of Fe I and Si I lines for the F supergiants in our sample. When combined with the (lower resolution) observations of Cenarro et al. (2001) we estimate a classification accuracy of \pm 2 spectral sub-types for our current observations, from which we identify two A and four F stars (Tables 1 and 2).

The narrow line widths, forming a smooth progression from the OB supergiants, together with the pronounced N I absorption features (cf. Figs. 17-25 of Munari \& Tomasella 1999) clearly justifies at least a supergiant classification for the 6 stars in question. However, determination of the bolometric luminosity of A-G stars may be made directly via the strength of the O I $7774 \AA$ Alend; a relationship first identified by Merrill (1925). We make use of the most recent calibration of this relationship by Arellano Ferro et al. (2003; their Eq. (2)); which is calibrated from $M_{V}=+0.35$ to $-9.5 \mathrm{mag}$. The results are presented in Table 2, with four stars (W4, 12a, 16a and 265) apparently demonstrating absolute visual magnitudes in excess of the current calibration for the relationship. Consequently, we simply limit ourselves to the conservative statement that these stars have $M_{V} \sim-9.5\left(\log \left(L_{*} / L_{\odot}\right) \sim 5.7\right)$; placing them at the empirical Humphreys-Davidson limit for cool hypergiants (under the assumption of negligible bolometric correction). We further note that both the presence of chemically processed material at the stellar surface and/or infilling of the blend due to emission from a stellar wind will only cause a systematic underestimate of the true luminosity; such an effect is apparently observed for the YHG $\rho$ Cas. Therefore, we conclude the six A-F stars in $\mathrm{Wd} 1$ have luminosities that are at least directly comparable to 

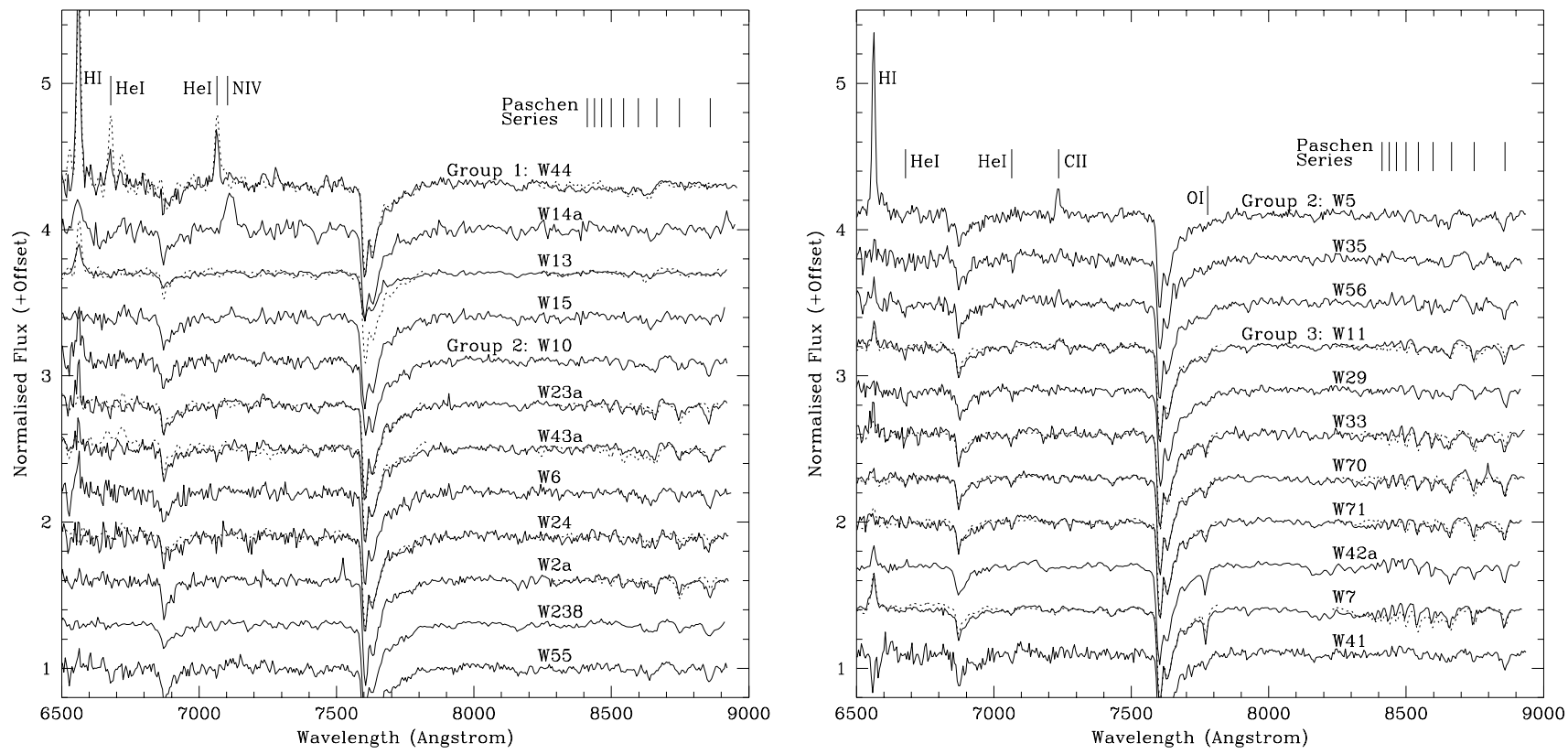

Fig. 7. Low resolution spectra of cluster OB supergiants (solid black lines). Where available high resolution spectra (from Figs. 4-6) are smoothed and overplotted (dotted red lines) on the corresponding low resolution spectra for comparison. Due to the low resolution an accurate determination of spectral type is impossible; hence we simply divide the spectra into 3 morphological classes based on the presence or otherwise of the Paschen lines and the O I $7774 \AA$ A feature; see Sect. 3.2 for further details.
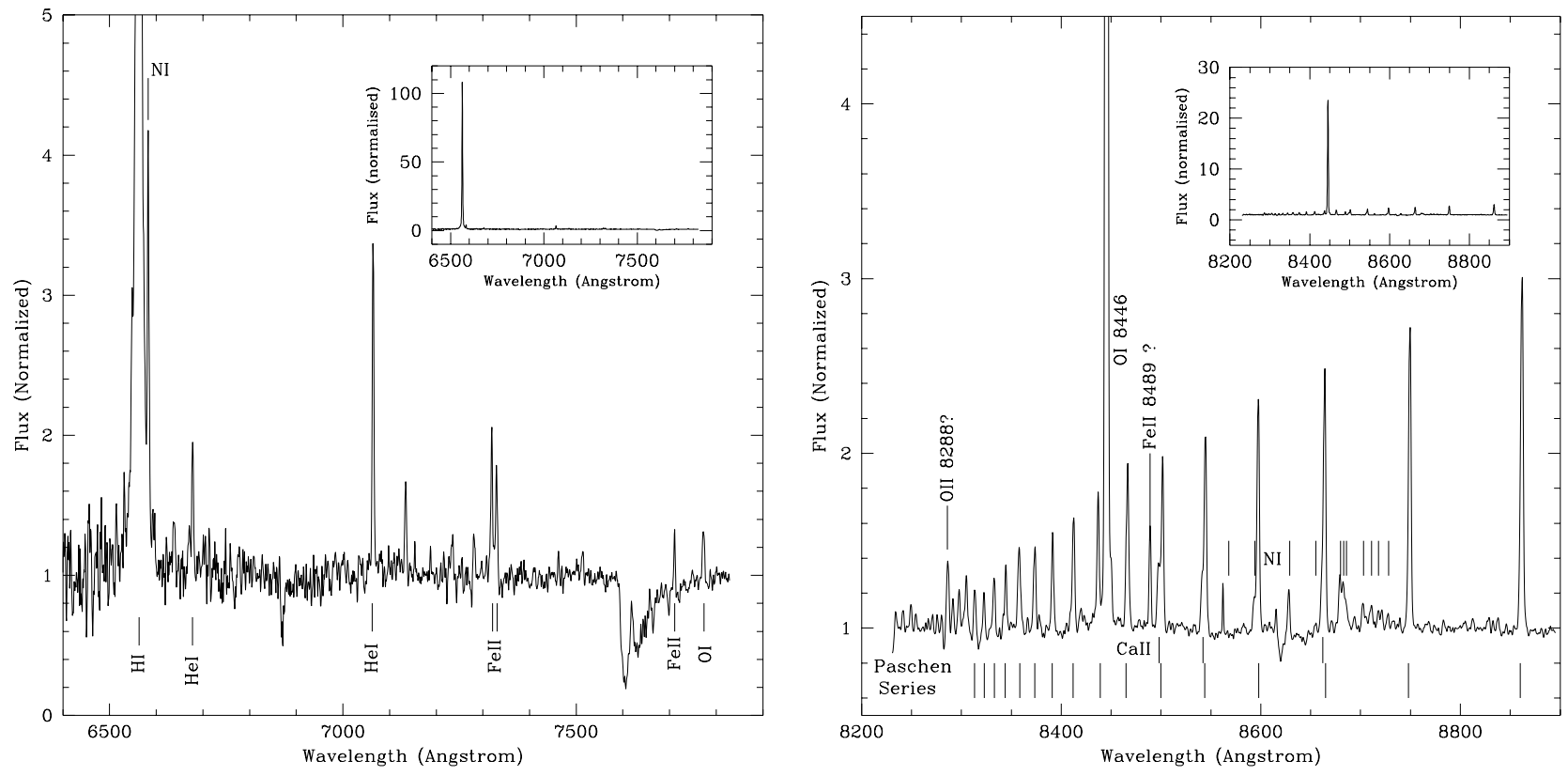

Fig. 8. $R$ and $I$ band spectra of the $\operatorname{sgB}[\mathrm{e}]$ star W9. Note that we are unable to identify the narrow emission features at 7134,7233 and $7280 \AA$.

those of the field populations of YHGs found in both the galaxy and the Magellanic Clouds.

However, as described by de Jager (1998) a high luminosity $\left(M_{V}>-7\right)$ is not sufficient to designate a star as a bona fide YHG. Rather, he defines the "Keenan-Smolinski" criteria for the spectroscopic classification of hypergiants; (i) the presence of one or more broad components of $\mathrm{H} \alpha$; and (ii) absorption lines that are significantly broader than those of Ia stars of similar spectral type and luminosity. Both criteria are designed to identify those stars with an enhanced mass loss rate, which de Jager (1998) introduces as the defining criteria of YHGs. Comparison of our spectra to those of bona fide YHGs demonstrates that their line widths are indeed consistent with such a classification (H. Nieuwenhuijzen priv. comm. 2003).

Between 2001-02, $\mathrm{H} \alpha$ has only been observed in emission in W16a, although West87 report emission in both W16a and W265, suggesting that it may be a transient phenomenon. Indeed, transitions from absorption to emission have been observed in $\rho$ Cas (Lobel et al. 2003), suggesting that the lack of $\mathrm{H} \alpha$ emission in the majority of the stars considered here may 

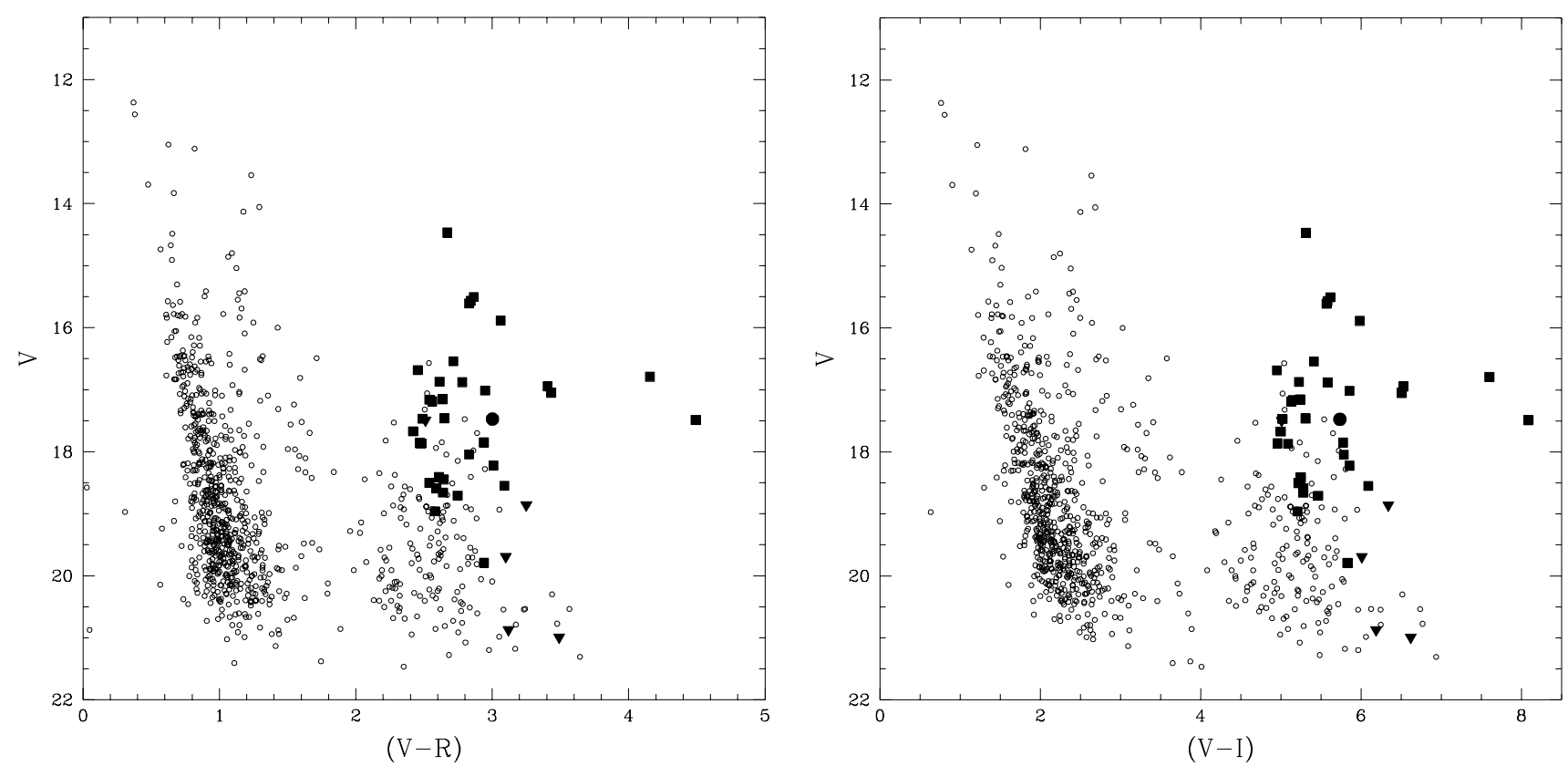

Fig. 9. Plot of $V$ band magnitudes versus the $(V-R)$ and $(V-I)$ colour indices for the $5.5 \times 5.5$ arcmin field centred on Wd 1 . Objects with spectroscopic classifications are indicated with filled symbols with red, yellow and blue squares for RSGs, YHGs and OB SGs respectively (the $\mathrm{sgB}[\mathrm{e}]$ star Wd 9 is indicated by a circle). WRs are indicated by magenta symbols, with squares WC and triangles WN stars; the 2 candidate WNL stars W5 and 44 are included in this category. Note that photometry is lacking for a number of cluster members such as the LBV W243, YHG W32, the RSG W20 and 6 WRs, while the two anomalously luminous OB SGs are the extreme B5 $\mathrm{Ia}^{+}$objects W7 and 33.

not disqualify them from a hypergiant classification. Moreover, we note that radio observations of the cluster (Dougherty et al. 2005) reveal all six to have radio counterparts which we suggest to be the result of the ambient UV radiation field provided by the hot star population of the cluster ionising their stellar winds (given that the stars themselves are likely too cool to ionise their own winds). We interpret this observation as an alternative diagnostic for mass loss from the stars and hence propose a hypergiant classification, noting that all six objects lie on the border or within the "yellow void", a region of the $\mathrm{H}$ $\mathrm{R}$ diagram occupied by known YHGs (e.g., Fig. 1 of de Jager 1998).

\subsection{The red supergiants; W20, 26 and 237}

Finally we turn to the three $\mathrm{M}$ supergiants within $\mathrm{Wd} 1$. Unfortunately, our current low resolution data do not allow us to improve on the classification of West87 for these objects. However, unlike West87, who concludes that W237 is seen in chance projection against $\mathrm{Wd} \mathrm{1,} \mathrm{we} \mathrm{suggest} \mathrm{that} \mathrm{it} \mathrm{is}$ in fact a bona fide member of the cluster. We base this conclusion not only on the comparable optical-mid-IR fluxes of the three $\mathrm{M}$ stars, but, more compellingly, the fact that all three are strong, spatially resolved radio sources (Dougherty et al. 2005). To the best of our knowledge no other RSGs have similar radio properties (e.g., Clark et al. 1998); hence we consider it likely that this phenomenon results from membership of $\mathrm{Wd} 1$, in a manner identical to that described above for the YHGs (Sect. 3.4).

The spectrum of W26 also demonstrates a strong double peaked emission line at $6564 / 82 \AA(E W=-74 \AA)$ that we attribute to a blend of $\mathrm{H} \alpha$ and [N II] $6583.5 \AA$ emission, noting that West87 also identifies $\mathrm{H} \alpha$ in the spectrum of W26 and W20. Two narrow emission lines at 9062 and $9525 \AA$ are also observed in the spectrum of W26. Owing to the difficulty of defining continua in these regions of the spectrum we have been unable to determine an $E W$ for either line, but tentatively identify them as [S III] 9069.4 and $9532.5 \AA$. W26 is known to be identified with extended radio and mid-IR emission (Clark et al. 1998) with an apparent bow shock morphology and we consider it likely that the emission arises in this nebular material. Identical emission lines are also observed towards two stars $\sim 1.5$ arcmin to the West (with colours consistent with membership of $\mathrm{Wd} 1$ ), which are also coincident with an extended region of radio emission (Dougherty et al. 2005). Thus we suggest a "nebular" rather than "stellar" origin for the emission lines, although we note that the nebular material associated with W26 likely results from the interaction of its stellar wind with the intracluster medium/wind.

\section{Photometric results}

In Fig. 9 we present $V$ vs. $(V-R)$ and $(V-I)$ colour magnitude plots for the 1044 stars within the $5.5 \times 5.5$ arcmin field containing $\mathrm{Wd} 1$, with photometric errors derived from DAOPHOT presented for BVRI bands in Fig. 10. Several distinct populations of stars are clearly delineated in both colour magnitude plots. Through comparison to the similar plot for the highly reddened cluster Pismis 23 (Piatti \& Claria 2002) we identify a long blue tilted Main Sequence (MS) for field stars from $V=12-21 \mathrm{mag}$ and $(V-R)=0.6-1.4$ and a vertical plume centred on $(V-R)=1.2$, which we identify 


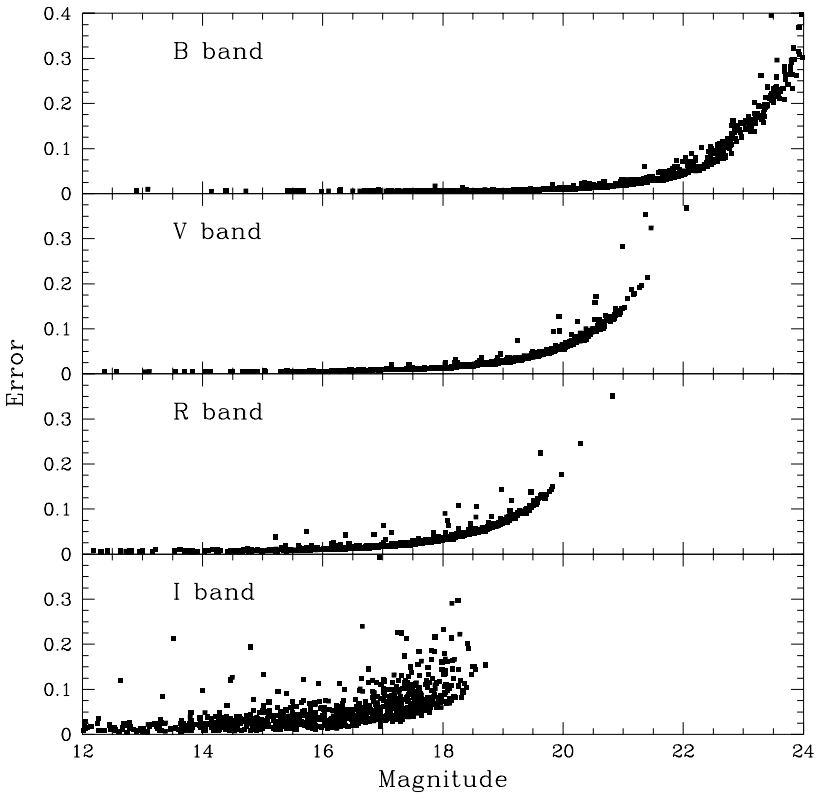

Fig. 10. Magnitude errors from DAOPHOT for the cluster field.

with a corresponding population of Horizontal Branch stars. A second, more sparsely populated MS is apparent at $(V-R) \sim$ $1.2-1.6$ - suggesting the presence of an intervening absorber ${ }^{3}$. Finally, we find a large population of highly reddened stars with $(V-R) \geq 2((V-I) \geq 4)$ that we propose as cluster members.

Photometry for those cluster members with spectroscopic identifications is provided in Table 1. Unfortunately photometry of 12 spectroscopically identified cluster members was unavailable due to severe blending or the star falling in the gap between the two detectors. The LBV W243 is one particularly regrettable example of the latter failing, as it had not been recognised as a variable star when the observations were made. In total we have currently identified 201 probable cluster members via a combination of spectroscopic and photometric observations.

\subsection{The reddening and distance to $\mathrm{Wd} 1$}

As Piatti et al. (1998), we find a scatter in the optical colours of cluster members that clearly exceeds the photometric errors, and implies significant differential extinction across the cluster. However, we note one essential difference between Piatti et al. and our results - we find no evidence for a Main Sequence in our current photometric data set. We may use our spectroscopically identified $\mathrm{OB}$ stars to study the reddening to $\mathrm{Wd} 1$ - given that the optical colours of all early OB supergiants are rather similar - finding no systematic gradients in reddening across the cluster. In Fig. 11 we plot the $(V-R)$ vrs. $(V-I)$ colours of the early and mid-OB supergiants to demonstrate the extent of the differential reddening. We find $E(V-R)$ and $E(V-I)$ to range from $\sim 2.6-3.2$ and $\sim 5.3-6.2$ with median values of 2.76

\footnotetext{
3 The photometric data of West87 - which were obtained for a greater field of view - reveal a much richer second MS, suggesting the obscuring cloud is of limited spatial extent, apparently covering a region not much larger than the cluster.
}

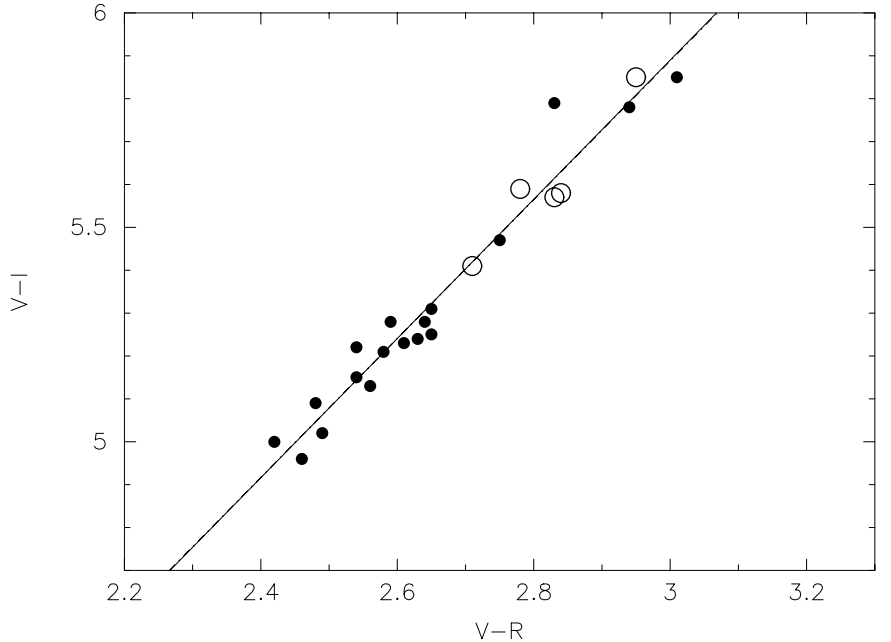

Fig. 11. Plot of the $(V-R)$ and $(V-I)$ colours for the 09.5 to $\mathrm{B} 0.5$ (filled symbols) and B3 to B5 supergiants (open), demonstrating the extent of the differential reddening across the cluster. Best fits to the reddening vector of the early and early+mid B supergiant datasets are also plotted and are found to be almost indistinguishable.

and 5.65 respectively ${ }^{4}$. We also plot least square fits to the data, allowing us to derive the reddening vector for the cluster. The resultant reddening vector does not pass through the locus for unreddened OB supergiants, indicating a non standard extinction law towards $W d 1$; a fact we return to below.

Given that a spectroscopic discriminant exists for the intrinsic luminosity of the YHGs, we may use these stars to determine $V-M_{V}$ to the cluster. Again, due to differential reddening we find this to range from 24-26.5 mag - the latter value for the isolated star W265 to the North of the cluster (noting a formal error of 0.4 mag due to the uncertainties in the calibration of the $M_{V}-E W_{\mathrm{OI} 7774}$ relationship). The mean and median values of $V-M_{V}$ are both $\sim 25.3 \mathrm{mag}$, which we adopt for the remainder of the paper. Piatti et al. (1998) derive a value of $V-M_{V}=23.8 \pm 0.3-$ at the lower range of values we find via isochrone fitting to the sequence of $\mathrm{OB}$ supergiants within Wd 1, which they mistook for a bona fide MS. They noted the difficulty of such a task given that their erroneous MS is both $\sim$ vertical and significantly broadened in Wd 1 due to differential reddening. Their vertical placement of the $4 \mathrm{Myr}$ isochrone was therefore accomplished via reference to the presence of the as then currently identified supergiants, for which Piatti et al. (1998) significantly underestimated their absolute visual magnitudes, leading to a corresponding underestimate of $V-M_{V}$.

Two other methods employed by Piatti et al. (1998) to infer $V-M_{V}$ - and hence the reddening and distance - for Wd 1 were comparison of their photometry to the values of $M_{V}$ quoted by West 87 for the brightest cluster members and a comparison to template clusters. The former suffered from the significant underestimation - by $>1$ mag on average - of the relevant $M_{V} \mathrm{~s}$

\footnotetext{
${ }^{4}$ Note that since the differential reddening is a real physical effect we present a range of values rather than a formal error derived from the standard deviation of the datatset and we choose to use the median rather than the mean value of colour excess since we find that latter to be skewed by a few stars with particularly high reddenings.
} 
by West 87 , while the latter invokes a mistaken comparison to the 10-15 Myr template open clusters NGC 457 and NGC 884; both cases leading to underestimates of $V-M_{V}$.

Adopting $V-M_{V} \sim 25.3$ mag to Wd 1 clearly demonstrates why no MS objects have yet been spectroscopically identified. An $07 \mathrm{~V}$ star (Sect. 5.1) with an absolute magnitude of $V=$ -4.7 would have an apparent magnitude of $V \sim 20.6 \mathrm{mag}$, at the limits of detectability for our current photometry and beyond the reach of our current spectroscopy. Consequently, we are led to the conclusion that the majority of cluster members currently identified are post-Main Sequence objects.

Finally, we may attempt to determine the distance to the cluster by individually dereddening the YHGs, for which we have a spectroscopic luminosity discriminant (which is lacking for the OB supergiants). This yields a mean distance of $\sim 5.5 \mathrm{kpc}$ and a mean $A_{\mathrm{v}} \sim 11.6 \mathrm{mag}$. We regard this distance estimate as an upper limit for three reasons. Firstly, increasing the distance would yield correspondingly larger luminosities for both the YHGs and RSGs, placing them above the empirical Humphreys-Davidson (HD) limit for cool stars. Indeed such a large distance - possibly biased by an overestimate of the luminosity of the isolated YHG W265 - already places W16a $\sim 0.5$ mag above the HD limit. Secondly, the distance determination assumes a standard reddening law which we know to be incorrect. From the median $E(V-I) \sim 5.65$ we find $A_{\mathrm{v}}=11.0$, via the ratio $E(V-I)=1.6 E(B-V)$ given by e.g., Fitzpatrick (1999) and $A_{\mathrm{v}}=3.1 E(B-V)$; appropriate for a standard reddening law. However, from the OB supergiants we measure a median $E(B-V) \sim 4.35$, resulting in an $A_{\mathrm{v}}=$ 13.6. Therefore we conclude that the above methodology results in an underestimate of the true reddening, leading in turn to an overestimate of the distance to $\mathrm{Wd} 1$. The final reason to favour a distance of $\leq 5.5 \mathrm{kpc}$ is that anything greater would place Wd 1 within $4 \mathrm{kpc}$ of the Galactic Centre, a region underpopulated by $\mathrm{H}$ II regions from which it might have formed (e.g., Fig. 5 of Russeil 2003).

A lower limit to the distance may be determined from the lack of radio detections of the Wolf Rayet population of $\mathrm{Wd} 1$ (Dougherty et al. 2005). Assuming a mass loss rate of $\sim 10^{-5} M_{\odot} \mathrm{yr}^{-1}$ and a wind velocity of $3000 \mathrm{~km} \mathrm{~s}^{-1}$ for the WRs, we would have obtained $3 \sigma$ detections even at a distance of $2 \mathrm{kpc}$ (Dougherty priv. comm. 2004). Consequently we adopt $2 \mathrm{kpc}$ as a lower limit to the distance of $\mathrm{Wd} 1^{5}$.

\subsection{The structure of Wd 1; spatial extent and mass segregation}

In Fig. 12 we present a plot of the positions of potential cluster members based on the colour cut offs above. Previous analysis by Piatti et al. (1998) had suggested that Wd 1 has a

\footnotetext{
5 We note that even this conservative estimate for the minimum distance to $\mathrm{Wd} 1$ is significantly in excess of the $1.1 \pm 0.4 \mathrm{kpc}$ finally adopted by Piatti et al. (1998). Such a low distance estimate resulted from the errors in determining $V-M_{V}$ described above and also the value adopted for the $E(B-V) / E(V-I)$ ratio. Specifically, Piatti et al. (1998) employ the calibration of Dean et al. (1978), while we have adopted the more recent calibrations of Rieke \& Lebofsky (1985) and Savage \& Mathis (1979), which have subsequently been confirmed by other studies (e.g., Cardelli et al. 1989; and Fitzpatrick 1999).
}

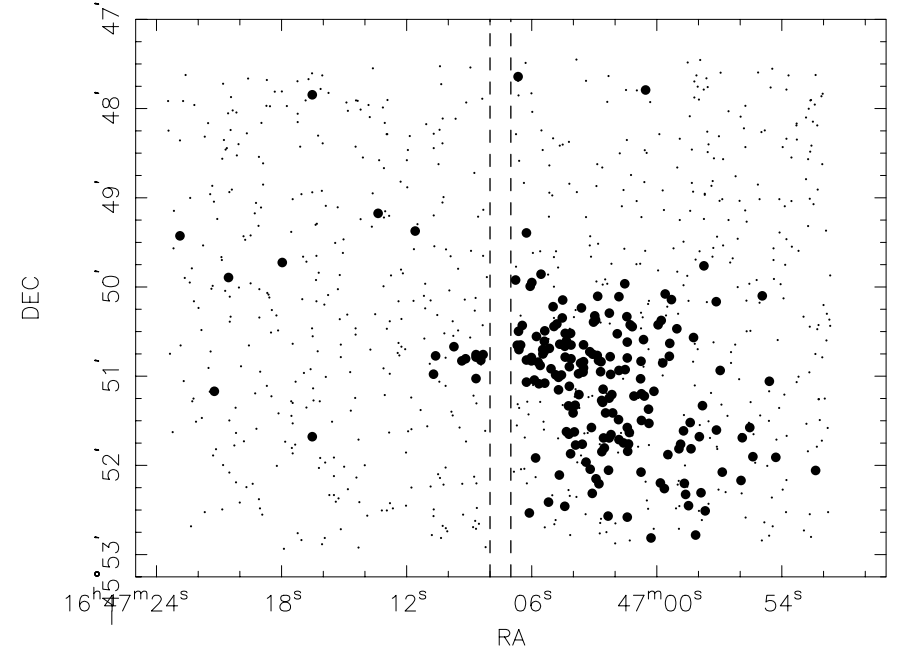

Fig. 12. Plot of the positions of the photometrically selected cluster members (solid circles) and field stars (dots). The position of the gap between the two CCDs is indicated by the dashed lines.

core+halo structure with radii of $\sim 36^{\prime \prime}$ and $\sim 72^{\prime \prime}$ respectively, with a radial light profile that diverges from that expected for a King profile. From Fig. 12 we find that $\sim 50 \%$ of the cluster members are found within a $\sim 50$ arcsec diameter "core", located approximately at RA = $164704 \delta=-440900$ (J2000), corresponding to $\leq 1.2 \mathrm{pc}$ for a distance of $\leq 5.5 \mathrm{kpc}$. An apparent deviation from spherical symmetry is present, with an overabundance of stars to the South West - however due to the requirement to site the cluster away from the gap between the CCDs we lack information on the extent of the cluster in this direction. To the North East we find a few candidate cluster members out to radii of $\geq 3$ arcmin (as measured from our cluster "core").

However, these values were determined solely from analysis of the distribution of the cluster supergiants, thus excluding both the more massive and evolved WR component, and also the currently undetectable Main Sequence $\left(M \leq 30 M_{\odot}\right.$; Sect. 5.1). Consequently, we refrain from drawing firm conclusions on the extent and cluster density profile. We do not expect the positions of the currently observed post-MS population to accurately represent the underlying stellar population, as many young clusters such as R136/NGC 2070 show evidence of strong mass segregation (e.g., Meylan 1993; Schweizer 2005). Indeed, by analogy with other young massive clusters we might expect the density profile of $\mathrm{Wd} 1$ to be described by a EFF (Elson et al. 1987) King-like profile (Schweizer 2005; Larsen 2004); this will be addressed in a future paper in which we analyse VLT (NAOS CONICA \& FORS1) imaging data.

\section{Discussion}

Spectroscopic and photometric analysis of the upper reaches of $\mathrm{Wd} 1$ reveal a unprecedented rich population of massive post-MS objects. Prior to this study, the three Galactic Centre clusters had hosted the richest population of such stars in the galaxy. In Table 3 we summarise the spectroscopic findings for Wd 1 and the populations of the Quintuplet, Arches and 
Table 3. Comparison of the stellar contents of $\mathrm{Wd} 1$ and the three Galactic Centre clusters (Figer 2005 and refs. therein). For brevity we define the "Early Transitional" category to encompass $\mathrm{sgB}[\mathrm{e}], \mathrm{LBV}$ and for Wd 1 the highly luminous mid-B supergiants $\mathrm{W} 7,33$ and 42 . The stellar content presented for $\mathrm{Wd} 1$ is restricted to those objects for which we have spectral classifications; hence while we are likely complete for the cool transitional objects the photometric data suggest we are severely incomplete for both OB supergiants and WRs. We have explictly excluded the WNL/B Ia+ star W5 and the WNE star possibly present as a blend in the W14a spectrum from the count of WR stars in Wd 1. Note that the WC content of the Quintuplet includes the five Quintuplet Proper Members, while for the Galactic Center cluster Genzel et al. (2003) propose 6 stars with Of/LBV classifications which we include as early transitional objects - hence the apparent absence of any OBIa stars for this cluster.

\begin{tabular}{ccccccc}
\hline \hline & OBIa & $\begin{array}{c}\text { Early } \\
\text { trans. }\end{array}$ & YHG & RSG & WN & WC \\
\hline Wd 1 & $\geq 25$ & $\geq 5$ & 6 & 3 & $\geq 7$ & $\geq 6$ \\
Quintuplet & 14 & 2 & 0 & 1 & 5 & 11 \\
Arches & 20 & 0 & 0 & 0 & $\geq 6$ & 0 \\
Center & - & 6 & 0 & 2 & $\geq 10$ & $\geq 10$ \\
\hline
\end{tabular}

Galactic Center clusters. Clearly, Wd 1 rivals, if not exceeds the stellar content of each of these clusters. While OB supergiants and WRs are found in all four clusters, a rich population of cool transitional objects is unique to $\mathrm{Wd} \mathrm{1}$; indeed, the population of YHGs in Wd 1 is equal to that found for the Galaxy or the LMC. Given the apparent brevity of this evolutionary phase, inferred from the rarity of these stars, such a population suggests that we are still incomplete for both their likely progenitors - OB supergiants - and descendants - WR stars.

Comparison to the current photometric dataset supports this conclusion. Assuming an average $M_{V} \leq-7$ and $V-M_{V} \sim$ 25.3 mag yields a total of 23 candidate OB supergiants with $V \leq 18$ mag (excluding known cool transitional objects); we have spectroscopically confirmed the identity of only $\sim 60 \%$ of this sample. Moreover, we note that a further 12 of the spectroscopically confirmed OB supergiants either lack photometry due to blending in the crowded "core" regions or are fainter than $V \sim 18$ - the faintest being W15, with $V \sim 19$ mag. Between $V=18-19$ mag we have spectroscopy of only 10 of 40 potential $\mathrm{OB}$ supergiants, although we note that an accurate derivation of a limiting magnitude for supergiant candidates will necessarily be a function of the significant differential reddening across $\mathrm{Wd} 1$.

Moreover, we expect that a proportion of the stars found between $V=18-20$ mag will likely be post-MS but of lower luminosity classes - Ib or II - and hence intrinsically fainter than the Ia stars identified to date. Given the limitations of the current spectroscopic data set, we cannot yet test this hypothesis, but we suggest that the the range in $V$ band magnitudes observed for OB stars with apparently identical colours (and hence extinctions) supports a range of instrinic luminosities for these stars.

More extreme results are found for the WRs. Only two stars are found to have $V<18 \mathrm{mag}$, six from a total of 159 cluster members have $V>18 \mathrm{mag}$ and six are photometric non-detections. Moreover, with the exception of the WNE star W72, all are either WNL or WCL - if present WNE or WCE stars might be expected to be $\geq 2$ mag fainter in the optical - well below our current detection threshold.

We therefore conclude that the current spectroscopically identified post-MS population for $\mathrm{Wd} \mathrm{1,} \mathrm{while} \mathrm{complete} \mathrm{for}$ the cool transitional objects is significantly incomplete for cluster OB supergiants and WRs, ignoring the effects of possible binarity and the incompleteness of the current photometric dataset due to crowding. Indeed, under the assumption of a Main Sequence turnoff at $\sim 07$ V (Sect. 5.1; currently undetectable at $V \sim 20.6 \mathrm{mag}$ ), a large majority of the currently identifiable cluster population will consist solely of post-MS objects.

\subsection{Progenitor masses for the cluster members}

Unfortunately, the current data do not permit the accurate construction of an HR diagram for $\mathrm{Wd} 1$. This is due to the difficulty in accurate spectral classification of the early OB supergiants from our present spectroscopic dataset. Currently we are unable to constrain the spectral types of the stars comprising this population to better than 09.5 to B0.5 Ia - or a range in temperature of $6000 \mathrm{~K}$, corresponding to an uncertainty in bolometric correction of $>0.6 \mathrm{mag}$. A similar problem is also present for the population of WRs and is further compounded by the lack of photometry for the majority of these stars. We further suspect that many of the WRs with detections are likely to be binary systems, a problem - compounded by blending - that also likely afflicts our sample of OB supergiants. Consequently, inferring stellar masses and a cluster age from the current dataset is difficult, particularly due to the lack of a detectable Main Sequence. Nevertheless, assuming co-evality - justified in Sect. 5.2 - we may make progress in determining likely masses for many of the cluster objects.

Trivially, the population of WRs are the initially most massive objects currently identified in Wd 1. From a study of 12 galactic clusters Massey et al. (2001) report that both WNL and WC stars are found in clusters with the highest turnoff mass $\left(\geq 50 M_{\odot}\right)$, although only one WCL star is reported in their study. By contrast, WNE stars are identified in clusters with a wide range of turnoff masses, with a lower limit of only $\sim 20 M_{\odot}$. Following the results of Massey et al. (2001) the currently identified WR population of Wd 1 - predominantly consisting of WNL and WCL stars - are likely descended from similarly high mass progenitors.

Of the transitional stars, comparison of the mean luminosity inferred for the YHGs $\left(\log \left(L / L_{\odot}\right) \sim 5.7\right)$ to theoretical predictions (e.g., Meynet \& Maeder 2003) suggests progenitor masses of $\sim 40 M_{\odot}$. This is consistent with the observation that galactic YHGs occupy a rather narrow range of luminosities $\log \left(L / L_{\odot}\right) \sim 5.6-5.8$ (e.g., Smith et al. 2004) - and hence progenitor masses. In this work Smith et al. suggest a possible evolutionary sequence for such stars of:

$\mathrm{O} \mathrm{MS} \rightarrow \mathrm{BSG} \rightarrow \mathrm{RSG} \rightarrow \mathrm{YHG} \rightarrow \mathrm{WN} 9-11 \rightarrow \mathrm{WR}$. 
If this scenario is correct, we might also expect the remaining cool and hot transitional objects to share a similar mass and luminosity with the YHGs.

One possible objection to this hypothesis might be the apparent presence of only a single $\mathrm{LBV}$ in $\mathrm{Wd} 1$, a phase which is known to occur for stars in excess of $\log \left(L / L_{\odot}\right) \sim 5.4$. However, the harsh UV rich environment of Wd 1 is likely to be inimical to the long term survival of LBV ejecta, such that only long term monitoring may reveal further examples. Obvious candidates include the extreme B supergiants W7, 33 and 42a, the WNL stars W5 and 44 and BSGs W23a and 43a, which apparently show evidence for $\mathrm{H} \alpha$ variability between the 2 epochs of our observations.

Moreover, Smith et al. (2004) identify an apparent lack of known LBVs in the luminosity range occupied by the YHGs and suggest a novel explanation for this - that the effect of the bi-stability jump on the mass loss rate of post RSG stars evolving bluewards into the S Doradus instability strip is sufficient to generate a pseudo-photosphere, effectively suppressing the LBV phase for stars of $\sim 40 M_{\odot}$. Thus, the apparent lack of LBVs in this luminosity range may be a real feature of the evolutionary sequence for stars of this mass.

Finally, as described in Sect. 3.3.1 the presence of $\mathrm{H} \alpha$ infilling or emission in the population of $\mathrm{OB}$ supergiants suggests that they have a mass at the upper range $-\geq 30 M_{\odot}$ - of those observed by McErlean et al. (1999). Thus we arrive at a picture of the current observed population of stars within $\mathrm{Wd} 1$ having progenitor masses ranging from $30-40 M_{\odot}$ (the OB supergiants) through to $\geq 40 M_{\odot}$ (the WRs), with the various cool (YHGs \& RSGs) and hot (LBV and extreme B supergiants) transitional objects defining a narrow range around $\sim 40 \mathrm{M}_{\odot}$. Assuming the OB supergiants represent the least evolved members of Wd 1 currently identified, a Main Sequence turnoff is likely to be found around $\sim 30-35 M_{\odot}$, implying an $\sim$ O7 V classification. As previously described, such a population would be below our current detection limit, thus consistent with the lack of any Main Sequence objects in our current photometric or spectroscopic data.

\subsection{The age of Wd 1}

While we may not determine an age for $\mathrm{Wd} 1$ on the basis of ioschrone fitting, we note that the presence of the WCL population suggests a minimum age of $\sim 3.5 \mathrm{Myrs}$, while the presence of $\mathrm{O}$ supergiants implies a maximum age of $\sim 5 \mathrm{Myr}$ (Meynet $\&$ Maeder 2003). The high luminosity and hence mass inferred for the YHGs suggest an age of $\sim 4$ Myrs (Clark \& Negueruela 2002, and refs. therein), while following Figer et al. (2002) the presence of relatively cool emission line B supergiants implies an age $\geq 4$ Myr. We therefore suggest a likely age for Wd 1 in the range 3.5-5 Myr, which is also consistent with our predictions of a MS turnoff around $\mathrm{O} 7 \mathrm{~V}$ or later.

An age in excess of $\sim 3 \mathrm{Myr}$ is supported by the lack of diffuse radio emission coincident with Wd 1 (e.g., Dougherty et al. 2005). It is expected that for clusters of this age a cluster superwind, driven both by the present stellar winds and also a possible supernova component, will have dispersed their natal
Table 4. Comparison of inferred properties of Wd 1 to other massive young clusters (after Table 5 of Figer et al. 1999). $M 1$ is the mass in observed stars and $M 2$ is the total mass assuming a Salpeter IMF between $1-120 M_{\odot}$.

\begin{tabular}{lcccc}
\hline \hline Cluster & $\begin{array}{c}\log M 1 \\
\left(M_{\odot}\right)\end{array}$ & $\begin{array}{c}\log M 2 \\
\left(M_{\odot}\right)\end{array}$ & $\begin{array}{c}\text { Radius } \\
(\mathrm{pc})\end{array}$ & $\begin{array}{c}\text { Age } \\
(\mathrm{Myr})\end{array}$ \\
\hline Wd 1 & 3.8 & 4.75 & 0.6 & $3.5-5$ \\
Quintuplet & 3.0 & 3.8 & 1.0 & $3-6$ \\
Arches & 3.7 & 4.3 & 0.19 & $2-3$ \\
Center & 3.0 & 4.0 & 0.23 & $3-7$ \\
NGC 3603 & 3.1 & 3.7 & 0.23 & 2.5 \\
R136 & 3.4 & 4.5 & 1.6 & $<1-2$ \\
\hline
\end{tabular}

envelopes. Examples of this phenomenon include the clusters Danks 1 and 2 found within the G305 star forming complex (Clark \& Porter 2004) and HD 32228 found in the LMC Giant H II region N11 (Walborn et al. 1999) ${ }^{6}$.

It is difficult to imagine a scenario in which $\mathrm{Wd} 1$ is not coeval, as is strongly suggested from the currently classified stellar population. The presence of just a few very massive stars would be expected to expel the gas not yet used in star formation from a cluster and halt further star formation on approximately a crossing time (e.g., Goodwin 1997). The lack of a significant gaseous component in Wd 1 strongly suggests that this has already happened. In order for $\mathrm{Wd} 1$ to be significantly non-coeval a mechanism would have to be found that formed only low-mass stars before forming all of the high-mass stars in a burst. Even if this were the case, our assumption of coevality for the observed high-mass stars would still be be correct.

\subsection{The mass of $W d 1$}

Currently, we have spectroscopically identified $\sim 50$ stars with progenitor masses $\geq 30 M_{\odot}$ within Wd 1 , placing an absolute minimum mass of Wd 1 at $1.5 \times 10^{3} M_{\odot}$. Under the assumption that all currently identified cluster members have masses of $\geq 30 M_{\odot}$ we derive a total mass of observed stars some $\sim 4$ times larger. Following Table 5 of Figer et al. (1999), in Col. 2 of Table 4 we compare the total mass of observed stars in Wd 1 to those of other young compact clusters within the Local Group. With the exception of the Arches and R136 clusters, we find that $\mathrm{Wd} 1$ is significantly more massive.

However, such a naked comparison takes no account of the either the depth of the relative observations, nor the effect that the different ages of the Arches and Wd 1 play. Specifically,

\footnotetext{
${ }^{6}$ We note that a second generation of trigged star formation is associated with both the G305 and N11 complexes. Examination of both radio (Dougherty et al. 2005) and mid-IR (Midcourse Space Experiment $8-25 \mu \mathrm{m}$ ) data reveals no such star formation activity associated with $\mathrm{Wd} 1$; hence we may infer a lack of significant remnant natal material associated with $\mathrm{Wd} 1$. Motivated by the comments of the referee, we speculate that either $\mathrm{Wd} 1$ formed from the collapse of the entire natal molecular cloud (possibly implying a higher than average star formation efficiency) or that the superwind generated by the unprecedented population of massive stars within $\mathrm{Wd} 1$ completely dispersed the cloud remnants before a second generation of stars could form.
} 
at an age of only $\sim 2.5 \mathrm{Myr}$ the mass-luminosity relationship is monotonic for the Arches, resulting in the mass estimate M1 being complete for stars $\geq 20 M_{\odot}$ (e.g., Serabyn et al. 1998). In contrast we have shown that our current observations are only complete for stars with initial masses $\geq 30 M_{\odot}$ in $\mathrm{Wd} 1$. Moreover, at an age of $\sim 4 \mathrm{Myr}$, the mass-luminosity relationship is bimodal, such that stars in excess of $40 M_{\odot}$ will either have been lost to supernovae or will have evolved to the WR phase. Indeed, van der Hucht (2001) demonstrates that with the exception of the latest WNLs, WRs of both flavours have absolute visual magnitudes $M_{\mathrm{v}}>-5 \mathrm{mag}-$ so undetectable in our current data ${ }^{7}$. Thus, we believe that our current observations of $\mathrm{Wd} 1$ are only sensitive to stars in a narrow range of progenitor masses - specifically 30-40 $M_{\odot}-$ and thus may not be directly comparable to those of the young $\leq 3 \mathrm{Myr}$ clusters such as NGC 3603, the Arches and R136 presented in Table 4.

Following Figer et al. (1999) we present the total mass for each cluster in Table 4 assuming an identical Salpeter IMF, finding $\mathrm{Wd} 1$ to be a factor of $\sim 2$ larger than the next most massive cluster, R136. We note however, that the current observational position on the full IMFs of massive star clusters is unclear. Sirianni et al. (2000) find that R136 has a Salpeter IMF that turns-over at $\sim 2 M_{\odot}$, as compared to $0.5 M_{\odot}$ in the field and local star forming regions (see Kroupa 2002 for a review of the IMF). However, this has been disputed by Zinneker et al. (2005) who claim a Salpeter slope to at least $1 M_{\odot}$.

Moreover, Figer (2005 and references therein) suggest a significant deviation from a Salpeter IMF for the Arches, prompting a downwards revison of the total mass to $\sim 10^{4} M_{\odot}$. The high gas temperatures (even in dense molecular clouds) due to the strong ambient radiation field in the Galactic Centre may well be responsible for this difference from the "standard" IMF and so the Galactic Centre clusters may not be directly comparable to Wd 1 or R136 which have formed in more "normal" environments. Indeed, Larsen (2004) finds that the massto-light ratios of SSCs are consistent with a Kroupa (2002) IMF. Another caveat is that these mass estimates are generally based on the assumption of virial equilibrium which may be significantly wrong when dealing with very young clusters (Goodwin et al. 2005).

In summary, it is currently unclear what the form of the IMF of massive star clusters is, or indeed if it is universal. Sirianni et al. (2000) do find that the IMF in fields close to R136 is Salpeter to at least $\sim 1 M_{\odot}$. It may be that primordial and/or dynamical mass segregation acts to make the IMF dependent on the size of the region under consideration, and that abnormal IMFs are the result of only considering the cores of clusters.

Nevertheless, assuming that $\mathrm{Wd} 1$ has a Kroupa two-part IMF and that $\geq 140$ of the current candidate members have masses $>30 M_{\odot}-$ conservatively defined as the number of stars with $V<20 \mathrm{mag}$, excluding the small WR component then we find that its mass must be $>10^{5} M_{\odot}$. This estimates makes no account of incompleteness and should be regarded as a lower limit under the hypothesis of a standard IMF. Moreover,

\footnotetext{
7 We suggest that the photometric detections of a subset of the cluster WRs - predominantly the WCLs - may be a result of binarity.
}

the additional assumption that our current observations only sample the stellar population within a restricted $\left(\sim 30-40 M_{\odot}\right)$ mass range suggests that we may be significantly underestimating the total mass of $\mathrm{Wd} 1$.

A more reliable comparison would be between the number count of stars within the 30-40 $M_{\odot}$ range - corresponding to a mid O Main Sequence star. The Arches has $\sim 40$ stars in this mass range (Figer et al. 2002) versus $\sim 140$ for $\mathrm{Wd} 1$. If the IMFs are similar this suggests that $\mathrm{Wd} 1$ is at least $3 \times$ more massive than the Arches. Similarly, Massey \& Hunter (1998) report that R136 has 34 stars more massive than $30 M_{\odot}\left(M_{V}<\right.$ -4.5) within $1 \mathrm{pc}$ of the cluster core, and 117 within $10 \mathrm{pc}$ (the nominal size of the NGC 2070 cluster). This makes Wd 1 significantly more massive than R136 and at least comparable to (and probably more massive than) the NGC 2070 cluster at the heart of the 30 Dor complex. It should also be noted that the currently known extent of Wd 1 is significantly less than $10 \mathrm{pc}$.

Similar conclusions may also be drawn for Cyg OB2 and W49A, both of which have recently been suggested to host massive stellar populations. On the basis of near IR photometry Knodlseder (2000) proposed that $\sim 120$ O stars (or their descendants) are found within Cyg OB2, which, depending on the low mass cut off adopted, yields a total mass in the range of 4-10 $\times 10^{4} M_{\odot}$. However subsequent near-IR (Comeron et al. 2002) and optical (Hanson 2003) spectroscopic surveys were unable to support such a conclusion, with Hanson concluding that many of the candidate OB stars are likely to be field contaminants and hence that the total $\mathrm{O}$ star census is unlikely to exceed 100. Moreover, at a distance of $\sim 1.7 \mathrm{kpc}$ Knodlseder (2000) finds Cyg OB2 to have a radius of $\sim 30$ pc (with a half light radius of $\sim 6 \mathrm{pc}$ ), an order of magnitude greater than we currently infer for $\mathrm{Wd} 1$.

Similarly, Alves \& Homeier (2003) propose that $\sim 100 \mathrm{O}$ stars are found within the central $16 \times 16 \mathrm{pc}$ region of the giant $\mathrm{H}$ II region W49A. However, as with Cyg OB2, the stellar density appears to be significantly lower than that inferred for $\mathrm{Wd} \mathrm{1,} \mathrm{with} \mathrm{only} \sim 30 \mathrm{O}$ stars found within the most massive cluster identified (Cluster 1 of Alves $\&$ Homeier, with a radius of $\sim 3 \mathrm{pc}$ ).

Therefore, despite the uncertainties in the appropriate IMF to apply, Wd 1 appears to be significantly more massive and compact than any other local massive star forming cluster (Fig. 13). Indeed, the only way in which to make Wd 1 comparable to, or less massive than, other local massive clusters would be to postulate that it has an IMF that turns-over at a very high mass $\left(5-10 M_{\odot}\right)$ so that very little mass is hidden in low-mass stars - arguably an equally important result.

The high mass and extremely compact nature of $\mathrm{Wd} 1$ make it a clear example of a SSC. Wd 1 is very probably more massive than the average old Galactic Globular Cluster $\left(10^{5} M_{\odot}\right)$, with a mass, luminosity and density that likely fulfills the criteria expected for proto Globular Clusters.

\subsection{Cluster dynamics and evolution}

At least half of the currently observed stellar population of $\mathrm{Wd} 1$ is found within a circular region of radius $\sim 25^{\prime \prime}$, corresponding to $\leq 0.6 \mathrm{pc}$ at $\leq 5.5 \mathrm{kpc}$. Assuming a mass of $>10^{5} M_{\odot}$ 


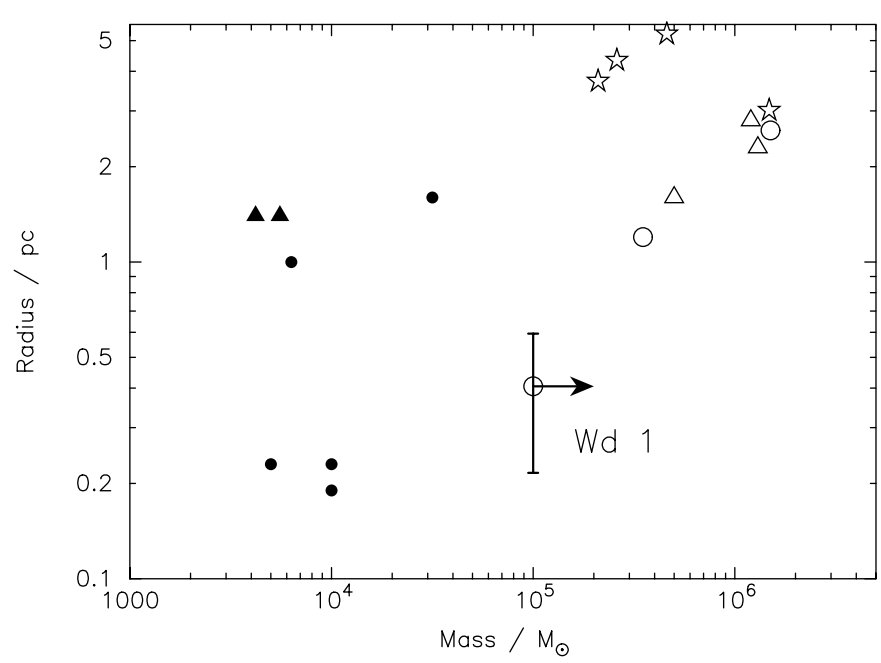

Fig. 13. Plot of the mass and radius of Wd 1 , selected young massive clusters in the Galaxy and LMC and SSCs for which dynamical estimates of their mass are available. Data from Figer et al. (1999; filled circles), Slesnick et al. (2002; filled triangles), Smith \& Gallagher (2001; open triangles), McCrady et al. (2003; open stars) and Larsen et al. (2004; open circles).

for Wd 1 leads to a minimum density of $\log \rho=5.0 M_{\odot} \mathrm{pc}^{-3}$, compared to $\log \rho=5.5 M_{\odot} \mathrm{pc}^{-3}$ found for the Arches cluster. Given that the distance is likely to be less than $5.5 \mathrm{kpc}$ and that this estimate does not account for incompleteness (which is expected to be worst in the centre of the cluster), we consider it likely that $\mathrm{Wd} 1$ is of comparable density to the Arches.

The collisional timescale for stars in the centre of $\mathrm{Wd} 1$ is of order $10^{4}$ Myr (Binney \& Tremaine 1987) assuming a central number density of stars of a few $\times 10^{6} \mathrm{pc}^{-3}$ for solar-type stars. This number will be significantly reduced by the presence of many large (in both mass and radius) stars (see for example the simulations of Portegies-Zwart et al. 2004). We might expect a collision between two of the stars in the core of $\mathrm{Wd} 1$ every $10^{3}-10^{4} \mathrm{yr}$, and so it is not unreasonable to propose that W9 is an unrelaxed recent merger remnant.

Recent work by Portegies-Zwart et al. (2004) suggest that under such extreme conditions runaway stellar mergers may lead to the production of an intermediate mass black hole. Accretion onto such objects has been suggested by some authors to explain the class of Ultraluminous X-ray Sources observed in external galaxies. If such an object is present in $\mathrm{Wd} 1$, the lack of such a luminous $\left(L_{\mathrm{x}}>10^{39} \mathrm{erg} / \mathrm{s}\right) \mathrm{X}$-ray detection (Clark et al. 1998) may indicate that either no mass donor is associated with it, or that mass transfer is either transitory or proceeds at a comparatively low rate (e.g., via direct wind fed accretion rather than Roche Lobe overflow).

Assuming a velocity dispersion in the core of $\sim 15 \mathrm{~km} \mathrm{~s}^{-1}$ (expected if the cluster core is approximately virialised) would imply that the core is $>100$ crossing times old. This would mean that the core is dynamically relaxed. It is possible that the outlying stars visible in Fig. 12 have been dynamically ejected from the core. Indeed, it is quite possible that some stars may have travelled in excess of $50 \mathrm{pc}$ from the cluster centre. Whilst the core of $\mathrm{Wd} 1$ should be dynamically relaxed, the outer regions are probably dynamically young and substructure in the initial cluster may not have been erased (e.g., Goodwin \& Whitworth 2004) as is thought to be the case in the NGC 2070 cluster (Meylan 1993).

The huge population of massive stars in $\mathrm{Wd} 1$ suggests that it will have a very significant impact on the local ISM. We estimate that the total number of type-II supernovae (SNII) will exceed 1500. This implies that Wd 1 will inject $>10^{55}$ erg of energy from stellar winds, UV radiation and SNII explosions on a timescale of $<40$ Myr. The energy flux from $\mathrm{Wd} 1$ will exceed $10^{40} \mathrm{erg} \mathrm{s}^{-1}$ for several Myr. The lack of radio emission and an HII region associated with Wd 1 (Sect. 5.2) suggests that Wd 1 already contains very little gas, presumably due to feedback from the massive stellar population. Indeed, the impact Wd 1 has already had upon its environment may be the reason why it has escaped identification as such an extreme cluster for so long.

The input of energy from many hundreds of SNII within Wd 1 would be expected to drive a significant superbubble and evacuate a large portion of the local Galactic disc (until destroyed by the differential rotation of the Galaxy). Wd 1 could well eject a significant amount of material out of the Galactic disc, driving a fountain or creating high velocity clouds.

$\mathrm{Wd} 1$ is also expected to return $>10^{3} M_{\odot}$ of heavy elements to the ISM (e.g., Tsujimoto et al. 1995). Due to the extremely high mass of many of the stars in Wd 1 compared to the content of lower-mass clusters, Wd 1 would be expected to return unusual abundances of heavy elements (e.g., Goodwin \& Pagel 2005).

\section{Conclusions}

We have presented both spectroscopic and photometric observations of the cluster Wd 1 . On the basis of their optical colours, we have identified $\sim 200$ candidate members, of which $95 \%$ are found to lie within 2 arcmin of the nominal cluster core, and $\sim 50 \%$ within an inner region of radius $\sim 25^{\prime \prime}$. Adopting an upper limit to the distance of $5.5 \mathrm{kpc}$ - from consideration of both spectroscopy and photometry - therefore implies that $\mathrm{Wd} 1$ is rather compact; the angular radii corresponding to $<2.9$ and $<0.6 \mathrm{pc}$ respectively.

We have obtained spectral classifications for 53 candidate cluster members, all of which are found to be post MS stars. Of these we find 14 to be WR stars of both flavours, 25 to be OB supergiants with spectral types of approximately B0 Ia, and at least 14 transitional objects. The latter group consists of both high (LBV, sgB[e] and extreme BSGs) and low (YHGs and RSGs) temperature objects. In doing this we confirm the result of West87 in identifying 4 YHGs within Wd 1 and add a further 2 examples, leading to a total population of such stars that is directly comparable to that of the Galaxy or the LMC.

Unfortunately, we are currently unable to construct an HR diagram for Wd 1. This is due to the uncertainties in the spectral classification of the OB supergiants, which preclude an accurate determination of their intrinsic luminosities, compounded by significant differential reddening across the cluster and the presence of a non standard extinction law. Nevertheless, we may use spectroscopic diagnostics to infer a mean luminosity for the YHGs of $\log \left(L / L_{\odot}\right) \sim 5.7$, placing them firmly 
at the HD limit and implying $\sim 40 M_{\odot}$ progenitors. Following the recent work of Smith et al. (2004) we infer similar initial masses for the other transitional objects within $\mathrm{Wd} 1$. While no unambiguous spectroscopic luminosity diagnostics exist for OB supergiants, following the results of McErlean et al. (1999) we infer progenitor masses of $\sim 30-40 M_{\odot}$ from the presence of $\mathrm{H} \alpha$ emission in their spectra.

These estimates suggest that we might expect a Main Sequence turnoff mass for $\mathrm{Wd} 1$ of around $30-35 M_{\odot}$, consistent with a population of late $\mathrm{O}$ dwarfs with spectral types no earlier than $\sim 07 \mathrm{~V}$. Adopting a median value for $V-M_{V} \sim$ 25.3 mag implies that such objects will be found at $V \sim$ 20.5 mag. This is entirely consistent with the lack of MS objects in our spectroscopic dataset, and the apparently lack of a MS in the photometric data. This in turn implies that a large majority of the $\sim 200$ currently identified cluster members are also likely to be post-MS objects.

Simply considering spectroscopically classified stars within $\mathrm{Wd} 1$, we find a minimum mass of the order of $1.5 \times$ $10^{3} M_{\odot}$. However, the twin assumptions that $\sim 140$ currently identified cluster members had initial masses $\geq 30 M_{\odot}$ and that a Kroupa (2002) two-part IMF is appropiate for Wd 1 yield a total cluster mass of $\sim 10^{5} M_{\odot}$. Note that this estimate takes no account of incompleteness in the current datasets nor that they are likely only sensitive to stars within the restricted $\sim 30-40 M_{\odot}$ range, both of which will act to increase the mass of the cluster. Nevertheless, Wd 1 appears significantly more massive than any currently identified open cluster in the Galaxy, and indeed is likely to be more massive than the average Galactic Globular Clusters. We conclude that Wd 1 represents the first known example of an SSC in the Local Group - i.e. a factor of 1000 times closer than the next closest SSC, NGC1569-A (Hunter et al. 2000).

With such an extreme mass and population of massive stars Wd 1 promises to greatly advance our understanding of the formation and evolution of massive stars, both individually, and in the extreme physical conditions present in SSCs. Given the wealth of post-MS objects, the identification of a MS and construction of an HR diagram will allow accurate progenitor masses to be assigned to different spectral types, permitting the refinement of current theoretical pathways through the post-MS "zoo" and the empirical verification of the HD limit for a population of co-eval stars at a single metallicity. Deep adaptive optics observation will permit the identification and study of sub-solar mass objects within $\mathrm{Wd} 1$ - if their formation has not been inhibited by the population of massive stars.

Indeed the presence of $\mathrm{Wd} 1$ within the galaxy will provide us with a unique insight into the physical processes occurring in the SSCs heretofore only identified in external starburst galaxies, challenging both our current theories of star formation and the role it plays in the wider evolution of the Galaxy. For example - assuming a nominal star formation efficiency of $\sim 10 \%-$ how did $\sim 10^{6} M_{\odot}$ of gas collect in a region $\sim$ one pc across? The average density of the $\mathrm{pc}^{3}$ molecular cloud from which Wd 1 formed must have been in excess of $10^{6}$ atoms $\mathrm{cm}^{-3}$ and hence the star formation process in this region must have been very different from that in local star forming regions such as Taurus or Orion. Recent simulations by Bonnell et al. (2004) of clustered star formation in a highly turbulent molecular cloud give probably the closest insight into star formation in $\mathrm{Wd} 1$.

Acknowledgements. We are indebted to the Peter Stetson for his help in reducing the photometric dataset. We thank the referee Nolan Walborn for his insightful comments and also thank Rens Waters, Sean Dougherty, Alex de Koter and Hans Nieuwenhuijzen for many informative discussions. We wish to thank ESO for their ongoing support for this research, and also the support astronomers at La Silla who have been of immense help. I.N. is a researcher of the programme Ramón y Cajal, funded by the Spanish Ministerio de Ciencia y Tecnología and the University of Alicante. This research is partially supported by the Spanish MCyT under grant AYA2002-00814. P.A.C. acknowledges financial support from the Royal Society. S.P.G. is a UKAFF fellow.

\section{Appendix A: OB supergiant classification criteria}

Caron et al. (2003) have recently outlined a classification scheme for O9 to B5 Ia-V stars between $\sim 8400$ to $8900 \AA$, based on both observations of standard stars and synthetic models. We have followed this methodology in the extension of this scheme to early $\mathrm{O}$ - late $\mathrm{B}$ supergiants, via the construction of an extended grid of non-LTE synethetic spectra, normalised where possible to observations of $\mathrm{OB}$ supergiants. We have further extended this to include the 6500-7900 ̊ range to encompass our full data set, although we concentrate on the $I$ band for our primary classification criteria.

We calculated our grid of OB supergiant models with the non-LTE atmospheric code CMFGEN (Hillier \& Miller 1998) between $T_{\text {eff }}=10 \mathrm{kK}$ and $T_{\text {eff }}=50 \mathrm{kK}$ at $2000 \mathrm{~K}$ intervals, accounting for line blanketing by $\mathrm{H}, \mathrm{He}, \mathrm{C}, \mathrm{N}, \mathrm{O}, \mathrm{Mg}, \mathrm{Al}, \mathrm{Si}, \mathrm{S}$, $\mathrm{Fe}$. Our approach follows the approach outlined for OB stars by Crowther et al. (2002), with an assumed $20 \mathrm{~km} \mathrm{~s}^{-1}$ microturbulence. A uniform rotational broadening of $75 \mathrm{~km} \mathrm{~s}^{-1}$ is adopted throughout.

The temperature scale for $\mathrm{O}$ supergiants has recently been revised downward as a result of the incorporation of line blanketing (Crowther et al. 2002; Herrero et al. 2003; Repolust et al. 2004). Similarly, the scale for early-B supergiants follows Crowther et al. (2005) whilst mid and late-B stars follow recent unpublished work by one of us (PAC). Luminosities for individual models follow from theoretical bolometric corrections, plus the Humphreys \& McElroy (1984) absolute magnitude-spectral type calibration.

We have adopted wind velocities for each subtype following Prinja et al. (1990) and Lamers et al. (1998), and massloss rates following the wind-momentum relationships from Kudritzki et al. (1999) for B stars ${ }^{8}$ and Puls et al. (1996) for O stars.

The synthetic spectra in the range 8200 to $8900 \AA$ are plotted in Fig. A.1 with, where available, standard spectra overplotted for comparison (the spectra of standard stars are summarised in Table A.1). A summary of the model parameters for each spectrum, along with optical and near IR colours

8 We adopted an identical form of the wind momentum relationship for late B supergiants to that established by Kudritzki et al. (1999) for mid-B supergiants. 

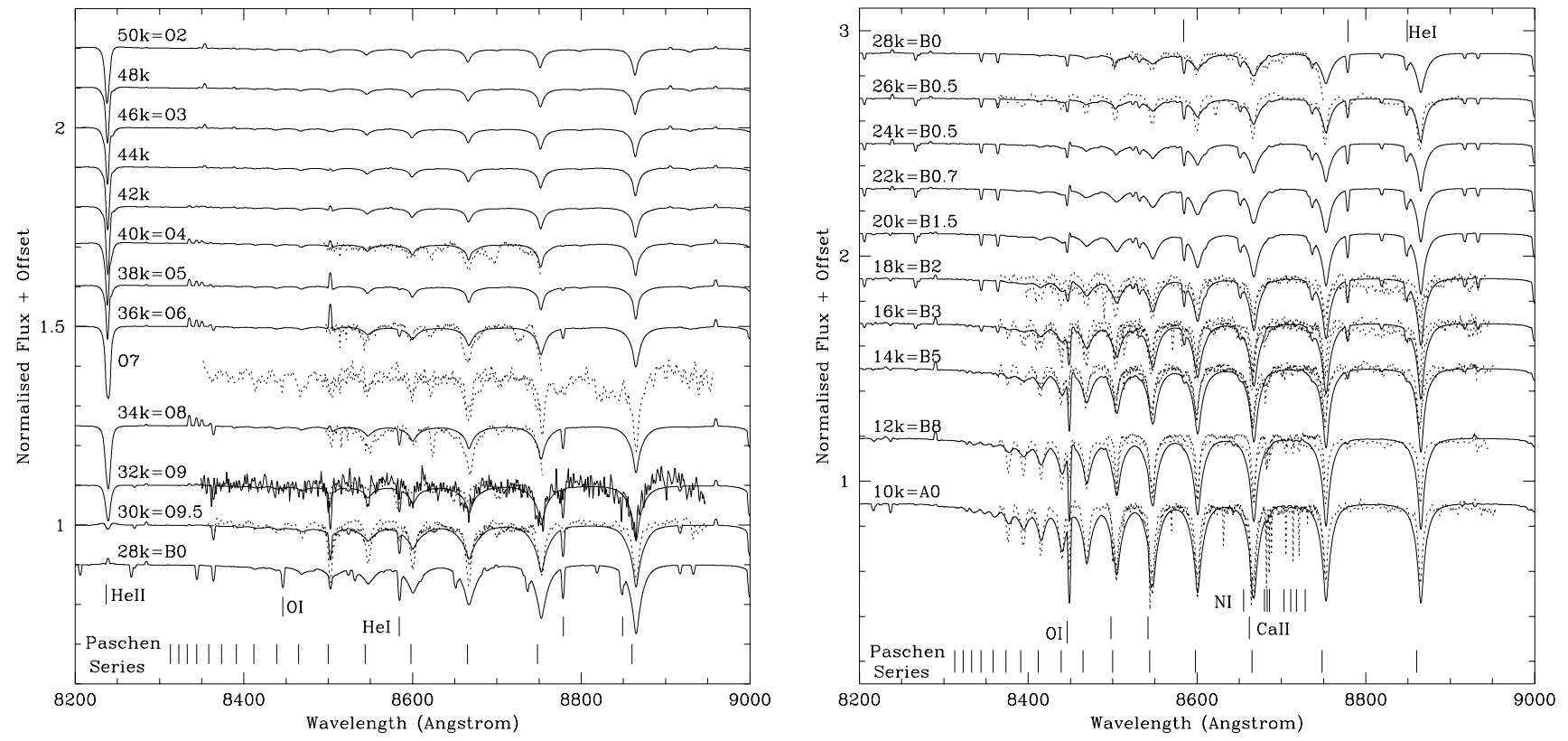

Fig. A.1. $I$ band ( 8200 to $9000 \AA$ ) synthetic spectra between 10 and $50 \mathrm{kK}$ (solid black lines; parameters given in Table 3). Prominent transitions of H I, He I, He II, O I, N I and Ca II indicated. Published OB supergiant spectra overplotted; data from Munari \& Tomasella (1999; red dotted lines), Cenarro et al. (2001; blue dotted lines) and Le Borgne et al. (2003; cyan dotted lines); stellar identification given in Table 2.

Table A.1. Individual standard stars used for the construction of Figs. 4-5. Data are from ${ }^{1}$ Munari \& Tomasella (1999), ${ }^{2}$ Le Borgne et al. (2003), and ${ }^{3}$ Cenarro et al. (2001). All stars are either type Ia or Ib supergiants, the latter given in italics.

\begin{tabular}{|c|c|}
\hline Spectral type & Star ID \\
\hline $\mathrm{O} 4$ & HD $190429^{1}$ \\
\hline O6 & HD $210839^{1}$ \\
\hline $\mathrm{O} 7$ & HD $192639^{1}$, HD $57060^{3}$ \\
\hline O8 & $H D 167971^{1}$ \\
\hline O9 & $H D 210809^{1}, H D 57061^{3}$ \\
\hline O9.5 & HD $228779^{1}$, HD $30614^{1}, H D 37742^{1}, H D 209975^{3}$ \\
\hline B0 & HD $37128^{1}$ \\
\hline B0.5 & HD $194839^{3}$ \\
\hline B2 & HD $268623^{2}$, HD $206165^{3}$ \\
\hline B3 & HD $198478^{1}$, HD $271163^{2}$, HD $14134^{3}$ \\
\hline B5 & HD $164353^{1,2}$, HD $13267^{3}$ \\
\hline B8 & HD $34085^{1}$, HD $199478^{3}$ \\
\hline A0 & HD $87737^{1}$, HD $39970^{3}$ \\
\hline
\end{tabular}

and $V$ band bolometric correction are presented in Table A.2. Comparison of synthetic spectra to observational data indicate an encouragingly close correspondance, given that models have not been tailored for the individual standard stars.

The first requirement of an $I$ band classification scheme for the current dataset is to distinguish between Main Sequence and Supergiant spectra, which Caron et al. (2003) demonstrate may be accomplished via the $F W H M$ of both the Paschen series and $\mathrm{He}$ I absorption features, which are systematically narrower for the supergiants due to the Stark effect (their Fig. 6). As a consequence, Paschen lines shortwards of Pa16 and the weak HeI transitions such as $8775 \AA$ are unresolvable in Main Sequence stars, while clearly present in supergiants of similar spectral type.

Accurate spectral classification of OB stars is more problematic, with Caron et al. (2003) forced to rely solely on the absolute strengths of the Paschen lines for classification of stars in the range O9-B5. While we too are forced to partially adopt this approach, particularly for stars with temperatures in excess of $34 \mathrm{kK}$ (O7I), we are able to identify a number of further temperature diagnostics in both standard and synthetic spectra. In attempting to describe a classification scheme we adopt the philosophy of relying on observational over synthetic data, and the occurrence of specific lines and particular line ratios rather than the absolute line strengths. For a line to be identifiable we require an $E W>0.2 \AA$ in either our synthetic or standard spectra. In practice all lines listed satisfy both criteria, with the exception of the Pa16+C III $\sim 8500 \AA$ blend, which is absent in our O8Ia $(34 \mathrm{kK})$ synthetic spectrum but clearly present in the standard spectra. He II $8238 \AA$ Aies outside the range of the standard spectra presented, but its occurrence is confirmed in unpublished $\mathrm{O}$ star spectra of one of us (Crowther).

Detailed criteria for spectral types $\mathrm{O} 2-\mathrm{A} 0$ are:

-O2 to O7 Ia (50 to $34 \mathrm{kK}$ ): the spectra of the earliest stars are dominated by the presence of He II $8238 \AA$ and Paschen series absorption lines alone. Consequently accurate spectral typing within this temperature range must rely solely on the relative strengths of the Paschen series, which our synthetic spectra indicate increases by a factor of $\sim 2$ between $\mathrm{O} 2$ to $\mathrm{O} 7$. Without adequate observations of standard stars to normalise our synthetic spectra to, we refrain from providing an absolute calibration of line strengths against spectral types (noting that fortuitously this does not affect our conclusions for $\mathrm{Wd} 1$ ). 
Table A.2. Stellar properties adopted for the construction of the grid of synthetic OB supergiant spectra presented in Fig. 3, presented with the resultant absolute $V$ band magnitude, $(V-I)$ and $(V-K)$ colour indices, and $V$ band bolometric correction (B.C.).

\begin{tabular}{ccccccccc}
\hline \hline $\begin{array}{c}T_{\text {eff }} \\
(\mathrm{kK})\end{array}$ & $\begin{array}{c}\text { Spec. } \\
\text { type }\end{array}$ & $\log \left(L / L_{\odot}\right)$ & $\begin{array}{c}\log \dot{M} \\
\left(M_{\odot} \mathrm{yr}^{-1}\right)\end{array}$ & $\begin{array}{c}v_{\infty} \\
\left(\mathrm{km} \mathrm{s}^{-1}\right)\end{array}$ & $\begin{array}{c}M_{V} \\
(\mathrm{mag})\end{array}$ & $\begin{array}{c}(V-I) \\
(\mathrm{mag})\end{array}$ & $\begin{array}{c}(V-K) \\
(\mathrm{mag})\end{array}$ & $\begin{array}{c}\text { B.C. } \\
(\mathrm{mag})\end{array}$ \\
\hline 50 & O2 & 6.24 & -4.80 & 3000 & -6.5 & -0.41 & -0.84 & -4.35 \\
46 & O3 & 6.13 & -4.92 & 2600 & -6.5 & -0.41 & -0.85 & -4.07 \\
40 & O4 & 5.95 & -5.18 & 2400 & -6.5 & -0.39 & -0.83 & -3.64 \\
38 & O5 & 6.02 & -5.09 & 2300 & -6.8 & -0.40 & -0.81 & -3.49 \\
36 & O6 & 5.94 & -5.20 & 2200 & -6.8 & -0.40 & -0.87 & -3.31 \\
34 & O8 & 6.04 & -5.08 & 2100 & -7.2 & -0.40 & -0.88 & -3.15 \\
32 & O9 & 5.97 & -5.18 & 2000 & -7.2 & -0.41 & -0.89 & -2.97 \\
30 & O9.5 & 5.89 & -5.26 & 1900 & -7.1 & -0.42 & -0.88 & -2.87 \\
28 & B0 & 5.75 & -5.8 & 1750 & -7.0 & -0.38 & -0.84 & -2.62 \\
26 & B0.5 & 5.69 & -5.9 & 1600 & -7.0 & -0.37 & -0.82 & -2.47 \\
24 & B0.5 & 5.60 & -5.9 & 1200 & -7.0 & -0.35 & -0.82 & -2.25 \\
22 & B0.7 & 5.52 & -6.0 & 1000 & -7.0 & -0.33 & -0.74 & -2.05 \\
20 & B1.5 & 5.43 & -6.9 & 800 & -7.0 & -0.32 & -0.70 & -1.83 \\
18 & B2 & 5.49 & -6.7 & 550 & -7.4 & -0.29 & -0.63 & -1.59 \\
16 & B3 & 5.30 & -6.9 & 400 & -7.2 & -0.22 & -0.55 & -1.30 \\
14 & B5 & 5.09 & -7.2 & 300 & -7.0 & -0.14 & -0.46 & -0.97 \\
12 & B8 & 4.93 & -7.4 & 250 & -7.0 & -0.05 & -0.34 & -0.57 \\
10 & A0 & 4.76 & -7.3 & 200 & -6.73 & +0.07 & -0.17 & -0.15 \\
\hline
\end{tabular}

-O8 to 09 Ia (34 to $32 \mathrm{kK})$ : while superficially similar, spectral types $\mathrm{O} 8$ to $\mathrm{O} 9$ may be distinguished from hotter $\mathrm{O}$ stars by the emergence of weak He I absorption features, most notably at $8775 \AA$. An additional diagnostic is the Pa16+C III blend at $\sim 8500 \AA$, which has a greater depth and strength than the adjacent Pa15 feature for O9 Ia stars.

-09.5 to B0 Ia (30 to $28 \mathrm{kK}$ ): O9.5 to B0 Ia spectra are characterised by the abrupt disappearance of He II absorption. As with O9 Ia stars, the Pa16+C III $\sim 8500 \AA$ A blend exceeds the strength of Pa15.

-B0.5 to B1.5 Ia (26 to $20 \mathrm{kK}$ ): spectra consist solely of $\mathrm{HI}$ and He I features; as with $\mathrm{O} 2$ to $\mathrm{O} 7$ Ia stars, detailed sub classification relies on the absolute strength of the Paschen series (cf. Caron et al. 2003 for a detailed analysis).

-B2 (18 kK): the appearance of the O I $8446 \AA$ line with a central intensity less than the adjacent Pa18 line acts as a diagnostic for B2 Ia stars.

-B3 (16 kK): as B2 Ia, but marked by the emergence of weak N I absorption features in the range $\sim 8670$ to $8730 \AA$.

-B5 (14kK): As above, but the intensity of O I $8446 \AA$ now exceeds that of Pa18.

-B8 to A0 (12 to $10 \mathrm{kK})$ : as B5 Ia but with the loss of residual He I absorption features, leaving a spectrum dominated by Paschen, O I and N I absorption.

Finally, an additional classification criterion found in the 6500 to $7900 \AA$ band is the OI $7774 \AA$ blend, which is detected $(E W>0.6 \AA)$ in both standard and synthetic spectra of spectral types of B0.7 and later. Therefore, if available, this additional diagnostic helps to break the degeneracy found for the 26 to $20 \mathrm{kK}$ (B0.5 to B1.5Ia) temperature range.

\section{References}

Alves, J., \& Homeier, N. 2003, ApJ, 589, L45

Andrillat, Y., Jaschek, C., \& Jaschek, M. 1995, A\&AS, 112, 475

Arellano Ferro, Giridhar S., \& Rojo Arellano, E. 2003, Rev. Mex. Astron. Astrofis., 39, 3

Bianchi, L., Bohlin, R., \& Massey, P. 2004, ApJ, 601, 228

Binney, J., \& Tremaine, S. 1987, Galactic Dynamics (PUP: Princeton), 541

Bonnell, I. A., Vine, S. G., \& Bate, M. R. 2004, MNRAS, 349, 735

Borgman, J., Koornneef, J., \& Slingerland, J. 1970, A\&A, 4, 248

Le Borgne, J.-F., Bruzual, G., Pelló, R., et al. 2003, A\&A, 402, 433

Cardelli, J. A., Clayton, G. C., \& Mathis, J. S. 1989, ApJ, 345, 245

Caron, G., Moffat, A. F. J., St-Louis, N., Gregg, A., \& Lester, J. B. 2003, AJ, 126, 1415

Carpay, J., de Jager, C., \& Nieuwenhuijzen, H. 1991, A\&A, 248, 475

Cenarro, A. J., Cardiel, N., Gorgas, J., et al. 2001, MNRAS, 326, 959

Clark, J. S., Fender, R. P., Waters, L. B. F. M., et al. 1998, MNRAS, 299, L43

Clark, J. S., Steele, I. A., \& Langer, N. 2000, ApJ, 541, L67

Clark, J. S., \& Negueruela, I. 2002, A\&A, 396, L25

Clark, J. S., \& Negueruela, I. 2004, A\&A, 413, L15

Clark, J. S., \& Porter, J. M. 2004, A\&A, 2004, 427, 840

Comeron, F., Pasquali, A., Rodighiero, G., et al. 2002, A\&A, 389, 874

Conti, P. S. 1976, Mem. Soc. R. Sci. Liège, 9, 193

Cotera, A. S., Erickson, E. F., Colgan, S. W. J., et al. 1996, ApJ, 461, 750

Crowther, P. A., \& Smith, L. J. 1999, MNRAS, 308, 82

Crowther, P. A., Hillier, D. J., Evans, C. J., et al. 2002, ApJ, 579, 774

Crowther, P. A., Lennon, D., \& Walborn, N. 2005, A\&A, submitted

Davidson, K., Gull, T. R., Humphreys, R. M., et al. 1999, AJ, 118, 1777

Draper, P. W., Taylor, M., \& Allan, A. 2000, Starlink User Note 139.12 , R.A.L. 
Dean, J. F., Warren, P. R., \& Cousins, A. W. J. 1978, MNRAS, 183, 569

de Grijs, R. 2005, in Proc. The Formation and Evolution of Massive Young Star Clusters, ed. H. J. G. L. M. Lamers, L. J. Smith, \& A. Nota, ASP Conf. Ser., 322, 29

de Jager, C. 1998, A\&AR, 8, 145

Dougherty, S. M., Clark, J. S., \& Waters, L. B. F. M. 2005, A\&A, in prep.

Elson, R. A., Fall, S. M., \& Freeman, K. C. 1987, ApJ, 323, 54

Falomo, R., Boksenberg, A., Tanzi, E. G., Tarenghi, M., \& Treves, A. 1987, MNRAS, 224, 323

Figer, D. F., McLean, I. S., \& Morris, M. 1999, ApJ, 514, 202

Figer, D. F., Najarro, F., Gilmore, D., et al. 2002, ApJ, 581, 258

Figer, D. F. 2005, in Proc. The Formation and Evolution of Massive Young Star Clusters, ed. H. J. G. L. M. Lamers, L. J. Smith, \& A. Nota, ASP Conf. Ser., 322, 49

Fitzpatrick, E. L. 1999, PASP, 111, 63

Genzel, R., Schodel, R., \& Ott, T. 2003, ApJ, 594, 812

Goodwin, S. P. 1997, MNRAS, 284, 785

Goodwin, S. P., \& Whitworth, A. P. 2004, A\&A, 413, 929

Goodwin, S. P. 2005, A\&A, in prep.

Goodwin, S. P., \& Pagel, B. 2005, MNRAS, submitted

Hanson, M. M. 2003, ApJ, 597, 957

Herrero, A. 2003, in A Massive Star Odyssey: From Main Sequence to Supernova, ed. K. van der Hucht, A Herrero, \& C. Estebanm (San Francisco: ASP), 2003, Proc. IAU Symp., 212, 3

Hillier, D. J., \& Miller, D. L. 1998, ApJ, 496, 407

Humphreys, R. M., \& McElroy, D. B. 1984, ApJ, 284, 565

Hunter, D. A., O'Connell, R. W., Gallagher, J. S., \& Smecker-Hane, T. A. $2000, A J, 120,2383$

Knoedlseder, J. 2000, A\&A, 360, 539

Koornneef, J. 1977, A\&A, 55, 469

Kroupa, P. 2002, Science, 295, 82

Kudritzki, R. P., Puls, J., Lennon, D. J., et al. 1999, A\&A, 350, 970

Landolt, A. U. 1992, AJ, 104, 372

Lamers, H. J. G. L. M., Snow, T. P., \& Lindholm, D. M. 1995, ApJ, 455, 269

Lamers, H. J. G. L. M., Zickgraf, F.-J., de Winter, D., Houziaux, L., \& Zorec, J. 1998, A\&A, 340, 117

Larsen, S. S., 2004, A\&A, 416, 537

Larsen, S. S., Brodie, J. P., \& Hunter, D. A. 2004, AJ, 128, 2295

Leitherer, C., Chapman, J. M., \& Koribalski, B. 1995, ApJ, 450, 289

Leitherer, C., Chapman, J. M., \& Koribalski, B. 1997, ApJ, 481, 898

Lobel, A., Dupree, A. K., \& Stefanik, R. P. 2003, ApJ, 583, 923

Lockwood, G. W. 1974, ApJ, 193, 103

Maeder, A., \& Conti, P. S. 1994, ARA\&A, 32, 22

Massey, P., \& Hunter, D. A. 1998, ApJ, 493, 180

Massey, P., DeGioia-Eastwood, K., \& Waterhouse, E. 2001, AJ, 121, 1050
McCrady, N., Gilbert, A. M., \& Graham, J. R. 2003, ApJ, 596, 240

McErlean, N. D., Lennon, D. J., \& Dufton, P. L. 1999, A\&A, 349, 553

Merrill, P. 1925, PASP, 37, 272

Meylan, G. 1993, in The Globular Cluster-Galaxy Connection, ed. G. H. Smith, \& J. P. Brodie (San Fransisco: ASP), ASP, 48, 588

Meynet, G., \& Maeder, A. 2003, A\&A, 404, 975

Munari, U., \& Tomasella, L. 1999, A\&AS, 137, 521 (MT99)

Negueruela, I., \& Clark, J. S. 2005, A\&A, submitted

Oudmaijer, R. D. 1998, A\&AS, 129, 541

Piatti, A. E., Bica, E., \& Claria, J. J. 1998, A\&AS, 127, 423

Piatti, A. E., \& Claria, J. J. 2002, A\&A, 388, 179

Prinja, R. K., Barlow, M. J., \& Howarth, I. D. 1990, ApJ, 361, 607

Puls, J., Kudritzki, R. P., Herrero, A., et al. 1996, A\&A, 305, 171

Portegies Zwart, S. F., Baumgardt, H., Hut Piet, Junichiro, M., \& McMillan, S. L. W. 2004, Nature, 428, 724

Repolust, T., Puls, J., \& Herrero, A. 2004, A\&A, 415, 349

Rieke, G. H., \& Lebofsky, M. J. 1985, ApJ, 288, 618

Russeil, D. 2003, A\&A, 397, 133

Savage, B. D., \& Mathis, J. S. 1979, ARA\&A, 17, 73

Shortridge, K., Meyerdicks, H., Currie, M., et al. 1997, Starlink User Note 86.15 , R.A.L

Schweizer, F. 2005, in Proc. The Formation and Evolution of Massive Young Star Clusters, ed. H. J. G. L. M. Lamers, L. J. Smith, \& A. Nota, ASP Conf. Ser., 322, 111

Serabyn, E., Shupe, D., \& Figer, D. F. 1998, Nature, 394, 448

Sirianni, M., Nota, A., Leitherer, C., De Marchi, G., \& Clampin, M. 2000, ApJ, 533, 203

Slesnick, C. L., Hillenbrand, L. A., \& Massey, P. 2002, ApJ, 576, 880

Smith, L. J. 2005, in Proc. The Formation and Evolution of Massive Young Star Clusters, ed. H. J. G. L. M. Lamers, L. J. Smith, \& A. Nota, ASP Conf. Ser., 322, 159

Smith, L. J., \& Gallagher, J. S. 2001, MNRAS, 326, 1027

Smith, N., Vink, J. S., \& de Koter, A. 2004, ApJ, 615, 475

Stetson, P. B. 1991, DAOPHOT User Manual

Tsujimoto, T., Nomoto, K., Yoshii, Y., et al. 1995, MNRAS, 277, 945 van der Hucht, K. A. 2001, NewAR, 45, 135

van Genderen, A. M., Bijleveld, W., \& van Groningen, E. 1984, A\&AS, 58, 537

Vreux, J. M., Dennefeld, M., \& Andrillat, Y. 1983, A\&AS, 54, 437

Walborn, N. R., Drissen, L., Parker, J. Wm., et al. 1999, AJ, 118, 1684

Walborn, N. R., \& Fitzpatrick, E. L. 2000, PASP, 112, 50

Westerlund, B. E. 1961, PASP, 73, 51

Westerlund, B. E. 1987, A\&AS, 70, 311 (West87)

Zinnecker, H. 2005, in Proc. IMF@50, eds. E. Corbelli, F. Palla, \& H. Zinnecker (Kluwer), in press 